\title{
Chemistry and distribution of daughter species in the circumstellar envelopes of O-rich AGB stars
}

\author{
Xiaohu Li ${ }^{1,2, \star}$, Tom J. Millar ${ }^{3}$, Alan N. Heays ${ }^{2}$, Catherine Walsh ${ }^{2}$, Ewine F. van Dishoeck ${ }^{2,4}$, and Isabelle Cherchneff ${ }^{5}$
}

${ }^{1}$ Key Laboratory of Optical Astronomy, National Astronomical Observatories, Chinese Academy of Sciences, A20, Datun Road, Chaoyang District, 100012 Beijing, PR China

e-mail: xiaohu@nao.cas.cn

2 Leiden Observatory, Leiden University, PO Box 9513, 2300 RA Leiden, The Netherlands

3 Astrophysics Research Centre, School of Mathematics and Physics, Queen's University Belfast, Belfast, BT7 1NN, UK

4 Max-Planck Institut für Extraterrestrische Physik (MPE), Giessenbachstr. 1, 85748 Garching, Germany

5 Departement Physik, Universitaet Basel, Klingelberstrasse 82, 4056 Basel, Switzerland

Received 26 January 2015 / Accepted 5 September 2015

\section{ABSTRACT}

\begin{abstract}
Context. Thanks to the advent of Herschel and ALMA, new high-quality observations of molecules present in the circumstellar envelopes of asymptotic giant branch (AGB) stars are being reported that reveal large differences from the existing chemical models. New molecular data and more comprehensive models of the chemistry in circumstellar envelopes are now available.

Aims. The aims are to determine and study the important formation and destruction pathways in the envelopes of O-rich AGB stars and to provide more reliable predictions of abundances, column densities, and radial distributions for potentially detectable species with physical conditions applicable to the envelope surrounding IK Tau.

Methods. We use a large gas-phase chemical model of an AGB envelope including the effects of $\mathrm{CO}$ and $\mathrm{N}_{2}$ self-shielding in a spherical geometry and a newly compiled list of inner-circumstellar envelope parent species derived from detailed modeling and observations. We trace the dominant chemistry in the expanding envelope and investigate the chemistry as a probe for the physics of the AGB phase by studying variations of abundances with mass-loss rates and expansion velocities.

Results. We find a pattern of daughter molecules forming from the photodissociation products of parent species with contributions from ion-neutral abstraction and dissociative recombination. The chemistry in the outer zones differs from that in traditional PDRs in that photoionization of daughter species plays a significant role. With the proper treatment of self-shielding, the $\mathrm{N} \rightarrow \mathrm{N}_{2}$ and $\mathrm{C}^{+} \rightarrow$ $\mathrm{CO}$ transitions are shifted outward by factors of 7 and 2, respectively, compared with earlier models. An upper limit on the abundance of $\mathrm{CH}_{4}$ as a parent species of $\left(\lesssim 2.5 \times 10^{-6}\right.$ with respect to $\left.\mathrm{H}_{2}\right)$ is found for IK Tau, and several potentially observable molecules with relatively simple chemical links to other parent species are determined. The assumed stellar mass-loss rate, in particular, has an impact on the calculated abundances of cations and the peak-abundance radius of both cations and neutrals: as the mass-loss rate increases, the peak abundance of cations generally decreases and the peak-abundance radius of all species moves outwards. The effects of varying the envelope expansion velocity and cosmic-ray ionization rate are not as significant.
\end{abstract}

Key words. astrochemistry - molecular processes - stars: AGB and post-AGB - circumstellar matter - stars: individual: IK Tau stars: mass-loss

\section{Introduction}

The asymptotic giant branch (AGB) phase occurs when stars from 0.8 to $8 M_{\odot}$ are undergoing their last nuclear burning (Iben \& Renzini 1983; Herwig 2005) and is a particularly interesting environment in which to study molecule formation and destruction. As shown in Fig. 1, the distribution and chemistry of species in the outer expanding circumstellar envelopes (CSEs), namely daughter species, are driven by hot (1000-2000 K) and shocked processes in the inner CSE where parent species are formed.

There are three types of AGB stars: $\mathrm{C}$-rich $(\mathrm{C} / \mathrm{O}>1)$, S-type $(\mathrm{C} / \mathrm{O} \approx 1)$, and $\mathrm{O}$-rich (M-type, $\mathrm{C} / \mathrm{O}<1)$. The largest variety of molecules is found in the CSE of C-rich stars, from simple CO to long carbon chains (e.g., Loup et al. 1993; Guélin et al. 1999; Cernicharo et al. 2000; Wakelam et al. 2010). The bestinvestigated AGB star, both observationally and theoretically, is the nearest C-rich star, IRC +10216 (e.g., Millar et al. 2000; Cernicharo et al. 2000; Woods et al. 2003; Cordiner \& Millar 2009; Decin et al. 2010a; De Beck et al. 2012; Cherchneff 2012;

^ East Asian Core Observatories Association (EACOA).
McElroy et al. 2013; Li et al. 2014). To date, more than $44 \%$ of the 180 identified species in the interstellar medium (ISM) or CSEs ${ }^{1}$ have been detected in this star, with some being first detections in astrophysical environments, e.g., the cyanide anion $\mathrm{CN}^{-}$(Agúndez et al. 2010b) and $\mathrm{FeCN}$ (Zack et al. 2011). A relatively small but increasing number of investigations have been made into S-type AGB stars (e.g., Ramstedt et al. 2006, 2009, 2011; Schöier et al. 2011; Danilovich et al. 2014), in which the central stars are undergoing evolution from an O-rich to a C-rich phase. During this transition, mixed chemistry may occur, especially when the O-rich material is stored in a stable disk around the central star, and features of both C-rich and O-rich chemistry may appear (Willems \& de Jong 1986; Szczerba et al. 2007). This interesting dual chemistry may continue to post-AGB stars, and even proto-planetary nebulae (pPNe; see Gielen et al. 2011, and references therein).

Significant progress in the investigation of O-rich CSEs has been driven by new telescopes (Ziurys et al. 2007; Tenenbaum et al. 2010; Decin 2012), especially the Heterodyne Instrument for the Far-Infrared (HIFI) on board the Herschel Space

1 http://www . astro.uni-koeln.de/cdms/molecules/ 


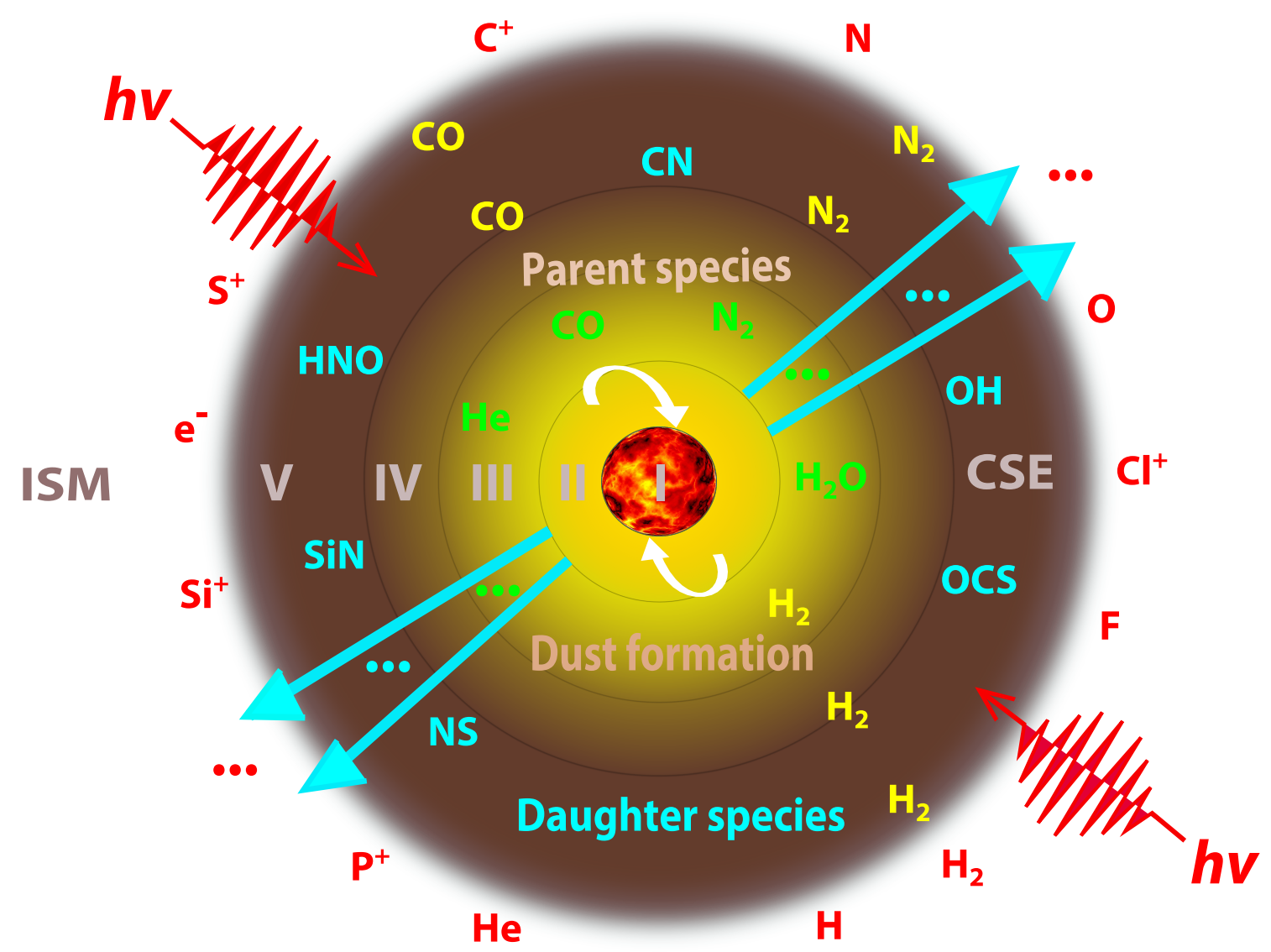

Fig. 1. Schematic structure of the CSE for an O-rich AGB star, which is divided into six regions for modeling purposes. (I): a degenerate C/O core and $\mathrm{He} / \mathrm{H}$ burning shell; (II): a convective atmosphere; (III): a shocked inner wind where some parent species (mainly $\mathrm{H}_{2} \mathrm{O}, \mathrm{CO}$, and $\mathrm{SiO}$ ) are formed; (IV): an intermediate expanding envelope where gas-phase chemistry may play a role; (V): an outer CSE where daughter species are formed primarily by photodissociation; (VI): the interstellar medium (ISM). This study focusses on the outer CSE where chemistry is mainly driven by the photodissociation and photoionization of molecules.

Observatory (Pilbratt et al. 2010), which provided velocityresolved spectra at far-infrared wavelengths that are not accessible from the ground. Several small molecules have been identified in O-rich evolved stars, for instance, $\mathrm{CO}, \mathrm{H}_{2} \mathrm{O}, \mathrm{HCN}$, HNC, CN, OH, SiS, SiO, and NO (e.g., Cho \& Ukita 1998; Ziurys 2006; Schöier et al. 2007; Ziurys et al. 2009; Maercker et al. 2009; Decin et al. 2010b; Quintana-Lacaci et al. 2013; Velilla Prieto et al. 2015). In addition, some O-bearing inorganic species, e.g., $\mathrm{AlO}$ and $\mathrm{AlOH}$, have been identified in the supergiant VY Canis Majoris (Tenenbaum \& Ziurys 2010).

Moreover, emission from polycyclic aromatic hydrocarbons (PAHs) and fullerenes $\mathrm{C}_{60}$ have also surprisingly been found in O-rich binary post-AGB sources (Gielen et al. 2011). All of these studies clearly show a rich inventory of molecules due to complex chemistry in the CSEs around O-rich central stars. The Atacama Large Millimeter/submillimeter Array (ALMA) is now starting to provide very sensitive, spatially resolved images of AGB envelopes as shown by the resolved shells of the AGB star R Sculptoris by Maercker et al. (2012). It is therefore appropriate to revisit the chemistry in these sources with an updated chemistry network.

In this work, we mainly focus on the well-observed O-rich AGB star, IK Tau (also known as NML Tau). This source has a large mass-loss rate of $\sim 4.5 \times 10^{-6} M_{\odot} \mathrm{yr}^{-1}$ (De Beck et al. $2010)$ in a wind that expands at a terminal velocity of $24 \mathrm{~km} \mathrm{~s}^{-1}$ (Justtanont et al. 2012). We note that these values are model dependent and may be different from those deduced from other observations (e.g., Maercker et al. 2008; Decin et al. 2010b). A few parent species (i.e., those formed close to the stellar photosphere) have been detected in the inner CSE of IK Tau in recent years, for instance, $\mathrm{CO}, \mathrm{HCN}, \mathrm{CS}, \mathrm{SiS}, \mathrm{SiO}, \mathrm{SO}$, and $\mathrm{SO}_{2}$ (Decin et al. 2010b; Kim et al. 2010), $\mathrm{H}_{2} \mathrm{O}$ (Decin et al. 2010c), $\mathrm{NH}_{3}$ (Menten et al. 2010), and PN and PO (De Beck et al. 2013). Schöier et al. (2013) conducted a survey of $\mathrm{HCN}$ in a sample of 69 stars that included all three types of AGB stars, including IK Tau, and concluded that the HCN abundances in C-rich AGB stars are, on average, around two orders of magnitude higher than those in O-rich stars. Fractional abundances of only two daughter species have been deduced from observations of IK Tau, CN (Decin et al. 2010b; Kim et al. 2010) and $\mathrm{HCO}^{+}$ (Pulliam et al. 2011). OH emission was identified but not quantified (Polehampton et al. 2010). In addition, thermal $\mathrm{CO}$ and $\mathrm{SiO}$ polarization has been mapped for IK Tau to study the orientation of its magnetic field (Vlemmings et al. 2012).

Willacy \& Millar (1997) built a model specifically for O-rich CSEs and discussed the chemistry of daughter species in four sources: R Dor, TX Cam, OH231.8 +4.2, and IK Tau. At that time, only limited observational data and a restricted chemical network were available; therefore, some assumptions made in that work have since been discounted, e.g., $\mathrm{CH}_{4}$ was assumed to be a parent species, but the observations by Marvel (2005) provided no evidence for $\mathrm{CH}_{4}$ based on sensitive searches for the chemically related $\mathrm{CH}_{3} \mathrm{OH}$ and $\mathrm{C}_{2} \mathrm{H}$ molecules. Agúndez et al. (2010a) employed an improved model to investigate the 
Table 1. Envelope parameters and assumptions for IK Tau in our fiducial model.

\begin{tabular}{ll}
\hline \hline 1. Shape & Spherical \\
2. Mass-loss rate & $4.5(-6) M_{\odot} \mathrm{yr}^{-1}$ (De Beck et al. 2010) \\
3. Envelope expansion velocity & $24 \mathrm{~km} \mathrm{~s}^{-1}(\mathrm{Justtanont}$ et al. 2012) \\
4. Stellar radius, $R_{*}$ & $2.1 \times 10^{13} \mathrm{~cm}$ (Duari et al. 1999) \\
5. Inner radius of CSE & $10^{15} \mathrm{~cm}$ \\
6. External radiation field & Standard interstellar radiation field (Draine 1978), isotropic incidence \\
7. Grain surface reactions & Ignored \\
8. Gas density distribution & Falls as $r^{-2}$, where $r$ is the distance from the central star \\
9. Chemical evolution & Kinetic equations solved as a function of radius as material traverses the CSE \\
10. $\mathrm{H}_{2}$ & Fully self-shielded, no photodissociation \\
11. Parent species & See Table 2 \\
12. $T, A_{V}$, and gas density & See Fig. 2 \\
13. Adopted cosmic-ray ionization rate of $\mathrm{H}_{2}$ & $1.2 \times 10^{-17} \mathrm{~s}^{-1}$ \\
\hline
\end{tabular}

Notes. $a(b)=a \times 10^{b}$.

chemistry in both O-rich and C-rich AGB stars, and suggested that the model predictions could be altered by taking into account a clumpy structure for the CSE, even with a simple approach. An improved list of parent species from observations was employed, but some parent species, e.g., $\mathrm{NH}_{3}$ and $\mathrm{HCN}$, had not yet been observed at that time, and therefore were not included as parents. The assumed abundances of parent species is very important in the simulation of outer CSE chemistry because these species trigger and drive the chemistry of daughter species. Previously, local thermodynamical equilibrium (LTE) predictions were used to determine the parent species. However, predictions from shock-induced non-LTE calculations (Duari et al. 1999; Cherchneff 2006) significantly differ from these; for example, Cherchneff (2006) concluded that four species (CO, SiO, $\mathrm{HCN}$, and CS) can exist in both $\mathrm{C}$-rich and O-rich stars. The conclusion was consistent with the previous observations (e.g., Bujarrabal et al. 1994) and was further confirmed via the observations of Decin et al. (2008).

In previous work we developed a model that, for the first time, accurately treated the photodissociation of two significant parent species, $\mathrm{CO}$ and $\mathrm{N}_{2}$, in the CSE of a C-rich star, IRC +10216 (Li et al. 2014). Here, we adapt this model to study the chemistry of O-rich AGB stars. The recent progress of both observations and models makes a detailed and more reliable simulation possible. The chemistry of a large number of potentially observable species, including $\mathrm{C}_{-}, \mathrm{N}-, \mathrm{O}-, \mathrm{Si}-, \mathrm{S}-, \mathrm{P}-, \mathrm{Cl}_{-}$, and F-bearing species are discussed.

The paper is organized as follows: the CSE model and improvements in this study are described in Sect. 2, followed by the results and discussion in Sect. 3, and conclusions in Sect. 4. A general investigation of the effects of varying the mass-loss rate and expansion velocity on the distributions of species is included in Appendix A.

\section{Methods}

\subsection{Improvements in the model}

The CSE model assumes that the gas expands in a smoothly expanding envelope with a constant mass-loss rate and velocity. The main assumptions of the model and envelope parameters of the O-rich AGB star, IK Tau, are listed in Table 1. More details about the CSE model can be found in McElroy et al. (2013), Millar et al. (2000), and Cordiner \& Millar (2009). The kinetic temperature of the gas, $T$, number density of molecular hydrogen, $n\left(\mathrm{H}_{2}\right)$, and visual extinction, $A_{V}$, are shown in Fig. 2 . The

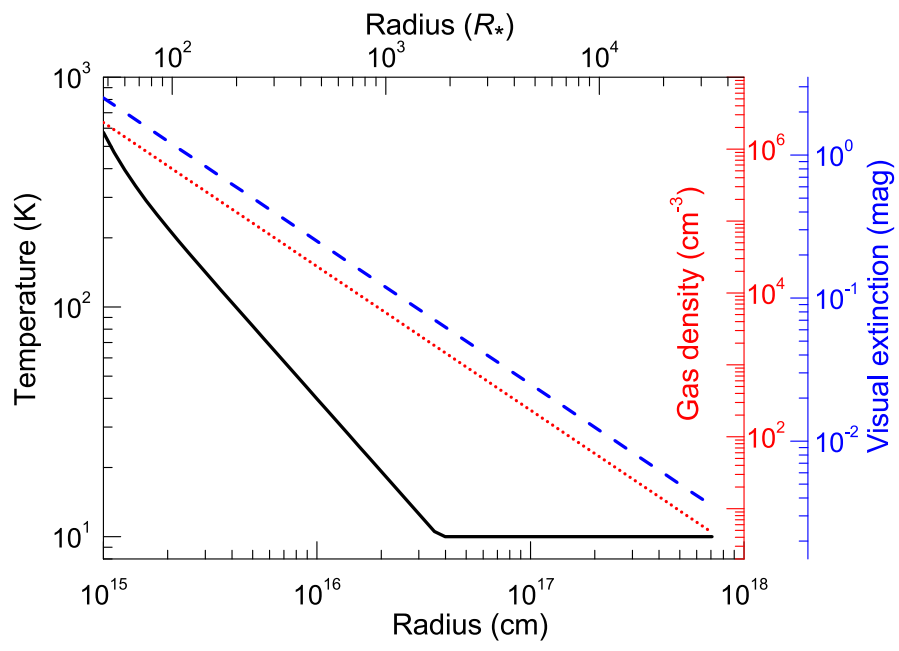

Fig. 2. Gas density, visual extinction, and gas temperature as a function of radius for the CSE of IK Tau from the center of the star towards the outside of the envelope. The radius is given in units of $\mathrm{cm}$ and stellar radii, $R_{*}$.

density follows a $r^{-2}$ behavior dictated by conservation of mass and the temperature structure is calculated according to the following relation enforcing a $10 \mathrm{~K}$ lower limit,

$T(r)=\max \left(100\left(r / r_{i}\right)^{-0.79}, 10\right)$,

where $r_{i}$ is the inner radius of our model. Extinction of the incident radiation is derived assuming purely absorbing grains and using standard values for the interstellar gas-to-dust ratio and extinction curve (Bohlin et al. 1978; Savage \& Mathis 1979).

The chemistry of daughter species in the outer CSE is dominated by photodissociation. Far-ultraviolet photons from all directions of the interstellar medium are assumed to contribute to the photodissociation rate of each species. IK Tau is of spectral type M6 with an effective temperature $\approx 2100 \mathrm{~K}$ (see, e.g, Duari et al. 1999); hence, the UV flux arising from the star itself is negligible. The model is assumed to be fully spherically-symmetric (SS model, Li et al. 2014) and accurately calculates the shielding effects of molecules in an isotropic interstellar radiation field.

The fifth release of the UMIST Database for Astrochemistry (UDfA, McElroy et al. 2013), hereafter RATE12, which contains 6173 gas-phase reactions involving 467 species, is further updated with a few new rate coefficients for the most significant nitrogen reactions (Wakelam et al. 2013) and is adopted in all 
of the calculations. Very minor differences (within $2 \%$ ) of the predicted peak abundances of the species are found when using these new rate coefficients compared with those using RATE12.

The treatment of $\mathrm{N}_{2}$ and $\mathrm{CO}$ photodissociation is the same as that described in our previous work (Li et al. 2014). Accurate photodissociation rates and shielding functions of $\mathrm{N}_{2}$ ( $\mathrm{Li}$ et al. 2013; Heays et al. 2014) are used. These data come from a concerted laboratory (e.g., Ajello et al. 1989; Helm et al. 1993; Sprengers et al. 2004; Stark et al. 2008; Lewis et al. 2008a; Heays et al. 2011) and theoretical (e.g., Spelsberg \& Meyer 2001; Lewis et al. 2005, 2008b; Ndome et al. 2008) effort over the last two decades. The CO photodissociation calculation uses the new rate and self-shielding functions from Visser et al. (2009). In the CSE model from (McElroy et al. 2013), the photodissociation rates of $\mathrm{N}_{2}$ and $\mathrm{CO}$ were adopted from van Dishoeck (1988), with self-shielding of CO treated only approximately (Morris \& Jura 1983). Photodissociation of $\mathrm{H}_{2}$ is not included in this work because it is fully self-shielded even at the largest radius considered in the model, for instance, the column density of $\mathrm{H}_{2}$ is $3.5 \times 10^{18} \mathrm{~cm}^{-2}$ at $7 \times 10^{17} \mathrm{~cm}$, corresponding to a shielding factor of $\sim 10^{-4}$ (Lee et al. 1996), which means photodissociation of molecular hydrogen is negligible. In our model reactions of atomic hydrogen are included, but generally they are unimportant because the fraction of $\mathrm{H}$ is usually small in regions in which chemistry is active. Other models that treat these processes explicitly show that atomic $\mathrm{H}$ starts to become significant $\left(n(\mathrm{H}) / n\left(\mathrm{H}_{2}\right)>10^{-4}\right)$ only in the very outer part of the envelope, beyond $3 \times 10^{17} \mathrm{~cm}$ (Glassgold \& Huggins 1983). Such a low atomic $\mathrm{H}$ fraction should not have a significant effect on the chemistry, although there could be very small effects at the outer edge due to charge transfer with $\mathrm{H}$, for example.

\subsection{New list of parent species}

The identification and abundance of parent species at inner radii is the first question to consider when modeling CSE chemistry. Typically three methods are used to constrain the parent species: TE predictions, shock-induced non-LTE predictions, and observations. The main benefit of theoretical predictions (TE and non-LTE) is that such models can provide a detailed list of all parent species. However, the disadvantage is that models are never as accurate as reality. Even assuming that the fundamental basis for the formation of these molecules is correct, the uncertainties in the physical processes in the inner CSE, chemical networks, and the initial abundances may still be significant. On the other hand, although high-quality data from powerful telescopes (e.g., Herschel, APEX, ALMA) provide direct constraints, some species are non-detectable. For example, $\mathrm{N}_{2}$, which is predicted to be the major elemental $\mathrm{N}$ reservoir in the inner layers; $\mathrm{N}_{2}$ is symmetric and thus possesses no electricdipole-allowed pure rotational spectrum. In addition, the identification of many species is not straightforward owing to the lack of detailed molecular data. Moreover, deduced results from spatially unresolved spectra are sometimes very model-dependent, which means considerable uncertainties also possibly exist.

Thanks to the recent progress in observations of O-rich AGB stars (e.g., Decin et al. 2010a,b,c; Kim et al. 2010; Menten et al. 2010; De Beck et al. 2013) and shock-induced non-LTE simulations (e.g., Cherchneff 2006), we are now able to employ an improved list of parent species (see Table 2) in this study. Wherever they are available, we employ the abundances of parent species from the latest high-quality observations, otherwise those from shock-induced non-LTE abundances are adopted.
Table 2. Initial fractional abundances $f_{0}(\mathrm{X})^{a}$ of parent species, relative to $\mathrm{H}_{2}$, for the O-rich AGB star, IK Tau.

\begin{tabular}{|c|c|c|c|}
\hline No. & Species & $f_{0}(\mathrm{X})$ & Comment \\
\hline 1 & $\mathrm{He}$ & $1.7(-1)$ & Solar abundance $^{b}$ \\
\hline 2 & $\mathrm{CO}$ & $2.0(-4)$ & Observation $^{c}$ \\
\hline 3 & $\mathrm{H}_{2} \mathrm{O}$ & $6.6(-5)$ & Observation $^{d}$ \\
\hline 4 & $\mathrm{~N}_{2}^{2}$ & $1.5(-4)$ & Shock-induced non-LTE abundance \\
\hline 5 & $\mathrm{NH}_{3}$ & $2.0(-6)$ & Observation $^{f}$ \\
\hline 6 & $\mathrm{HCN}$ & $4.4(-7)$ & Observation $^{c}$ \\
\hline 7 & $\mathrm{CO}_{2}$ & $4.4(-9)$ & Shock-induced non-LTE abundance \\
\hline 8 & $\mathrm{CS}$ & $8.0(-8)$ & Observation $^{c}$ \\
\hline 9 & $\mathrm{SiS}$ & $1.1(-5)$ & Observation $^{c}$ \\
\hline 10 & $\mathrm{SiO}$ & $1.6(-5)$ & Observation $^{d}$ \\
\hline 11 & SO & $2.0(-6)$ & Observation $^{d}$ \\
\hline 12 & $\mathrm{SO}_{2}$ & $2.0(-6)$ & Observation $^{c}$ \\
\hline 13 & $\mathrm{H}_{2} \mathrm{~S}$ & $1.0(-8)$ & Shock-induced non-LTE abundance \\
\hline 14 & HS & $2.3(-8)$ & Shock-induced non-LTE abundance \\
\hline 15 & PN & $3.0(-7)$ & Observation $^{g}$ \\
\hline 16 & $\mathrm{PO}$ & $9.0(-8)$ & Observation $^{g}$ \\
\hline 17 & $\mathrm{HCl}$ & $3.7(-7)$ & Shock-induced non-LTE abundance \\
\hline 18 & $\mathrm{HF}$ & $7.3(-7)$ & TE prediction ${ }^{h, b}$ \\
\hline
\end{tabular}

Notes. $a(b)=a \times 10^{b} .{ }^{(a)}$ For species $\mathrm{X}, f_{0}(\mathrm{X})=n(\mathrm{X}) / n\left(\mathrm{H}_{2}\right) .{ }^{(b)}$ Asplund et al. (2009). ${ }^{(c)}$ See Table 6 in Decin et al. (2010b). In that work the fractional abundance was given relative to the total H-content, $n_{\mathrm{H}}$, by assuming all hydrogen is in its molecular form, $\mathrm{H}_{2}$, i.e., $n_{\mathrm{H}}=2 n\left(\mathrm{H}_{2}\right)$. ${ }^{(d)}$ Decin et al. (2010c). ${ }^{(e)}$ Gobrecht et al. (2016). ${ }^{(f)}$ Menten et al. (2010). ${ }^{(g)}$ De Beck et al. (2013). ${ }^{(h)}$ TE prediction, assuming elemental F has the solar abundance.

Predictions from TE will only be used when no data are available from non-LTE or observational investigations. The effect of uncertainties in the abundances assumed for parent species is discussed in Sect. 3.12. We note that we do not simulate the chemistry in the intermediate envelope $\left(\lesssim 100 R_{*}\right)$ within which gas-phase chemistry may alter the abundances of parent species.

\section{Results}

\subsection{General chemistry}

Photochemistry induced by external UV photons dominates the chemistry in the expanding envelope of an AGB star. Generally, the formation and destruction processes of daughter species are controlled by the conversion $\mathrm{XY} \rightarrow \mathrm{X} \rightarrow \mathrm{X}^{+}$, as shown in Fig. 3 . A major finding in this work is that photoionization of molecules is also significant in the outer layers: $\mathrm{XY} \rightarrow \mathrm{XY}^{+}$. In this sense, the CSE chemistry of O-rich AGB stars differs from that found in standard photon-dominated region (PDR) models in which the ion-molecule chemistry of O-bearing species is driven by charge exchange reactions with abundant cations (see, e.g., Sternberg \& Dalgarno 1995; Jansen et al. 1995a,b).

The synthesis continues with $\mathrm{X}^{+}$(and $\mathrm{XY}^{+}$) abstracting $\mathrm{H}$ from $\mathrm{H}_{2}$ by ion-molecule reactions forming molecular hydride cations, which is frequently followed by dissociative recombination that leads to neutral molecular hydrides, and ends up in the formation of atoms or cations at the edge of the CSE. The model predicts that negative ions are not abundant in O-rich AGB stars.

\subsection{Impact of new $\mathrm{N}_{2}$ and $\mathrm{CO}$ photodissociation rates}

A proper treatment of the shielding of both $\mathrm{N}_{2}$ and $\mathrm{CO}$ photodissociation has a significant impact on the chemistry in the outer CSE of AGB stars. Among the various shielding effects, self-shielding is the most important factor in reducing the 


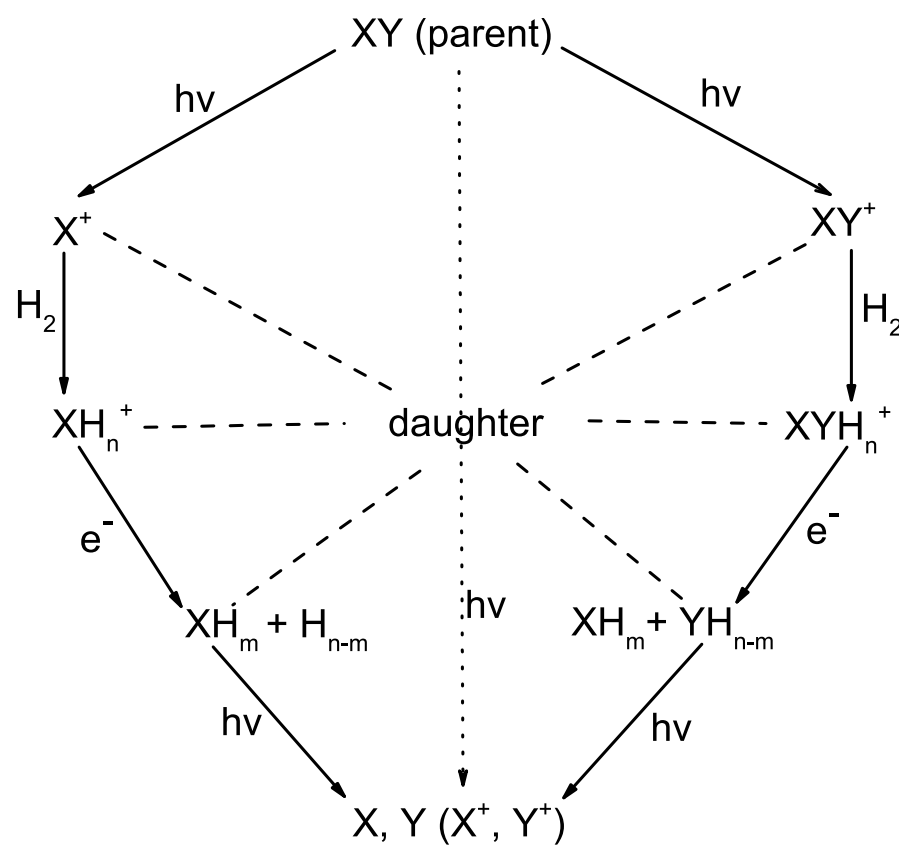

Fig. 3. General pathways for parent species giving rise to daughter species in the outer CSE of an O-rich AGB star.

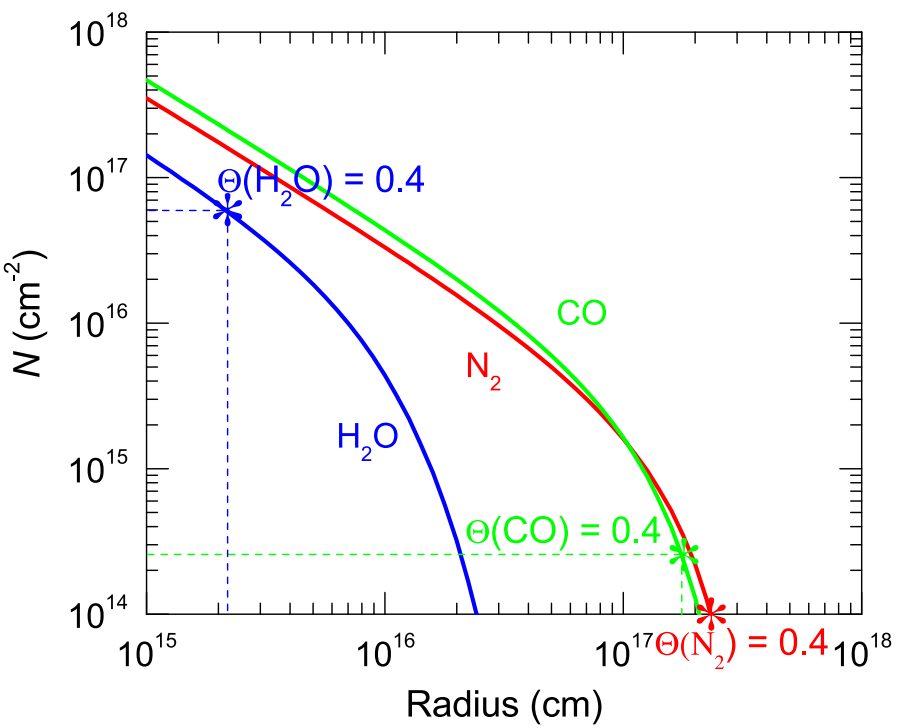

Fig. 4. Column densities of $\mathrm{H}_{2} \mathrm{O}, \mathrm{CO}$, and $\mathrm{N}_{2}$ as a function of radius. The asterisks indicate a value of the column density that corresponds to a self-shielding factor of 0.4 for these molecules. Shielding effects become considerable when the column densities are higher than those indicated.

photodissociation rate of $\mathrm{N}_{2}$ and $\mathrm{CO}$ ( $\mathrm{Li}$ et al. 2014). As shown in Fig. 4, self-shielding of $\mathrm{CO}$ and $\mathrm{N}_{2}$ becomes considerable at a large radius, around $2 \times 10^{17} \mathrm{~cm}$, where photodissociation from the external interstellar field is important. To accurately investigate the chemistry at smaller radii, we have considered full shielding (from dust, molecular $\mathrm{H}_{2}$, atomic $\mathrm{H}$, and the molecule itself) of both $\mathrm{N}_{2}$ and $\mathrm{CO}$ in all of our calculations.

Figure 5 shows that the location of the transition zone from $\mathrm{N}_{2} \rightarrow \mathrm{N}$ is shifted outwards by a factor of 7 when accurately considering shielding effects of $\mathrm{N}_{2}$ with the SS model, whereas the location for the conversion from $\mathrm{CO} \rightarrow \mathrm{C}^{+}$moves outwards by a factor of 2 when using updated shielding functions. These changes result in an important difference in the distributions of

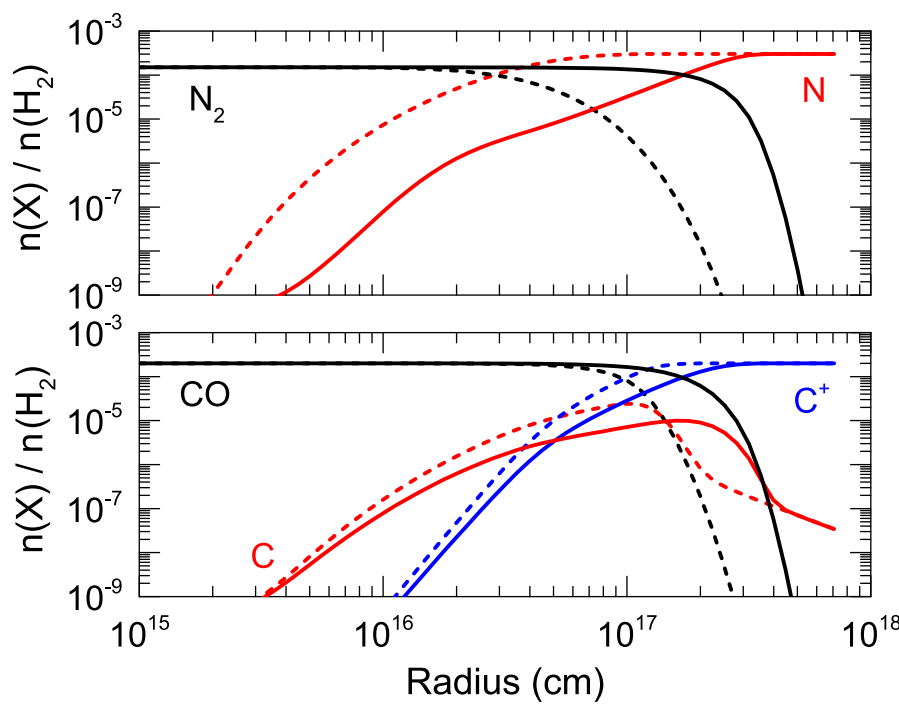

Fig. 5. Fractional abundances, relative to $\mathrm{H}_{2}$, of $\mathrm{N}_{2}$ and $\mathrm{N}$ (top panel) and $\mathrm{C}^{+}, \mathrm{C}$, and $\mathrm{CO}$ (bottom panel) as a function of radius. Solid lines: from the SS model ( $\mathrm{Li}$ et al. 2014), full shielding (dust + self- $+\mathrm{H}_{2}$ $+\mathrm{H}$ ) are considered using an updated $\mathrm{N}_{2}$ photodissociation rate and self-shielding function ( $\mathrm{Li}$ et al. 2013). Dashed lines: calculated from a model similar to that of McElroy et al. (2013), but adopting assumptions specifically for IK Tau. In this model only dust shielding is considered when calculating $\mathrm{N}_{2}$ photodissociation, and $\mathrm{CO}$ self-shielding is treated only approximately.

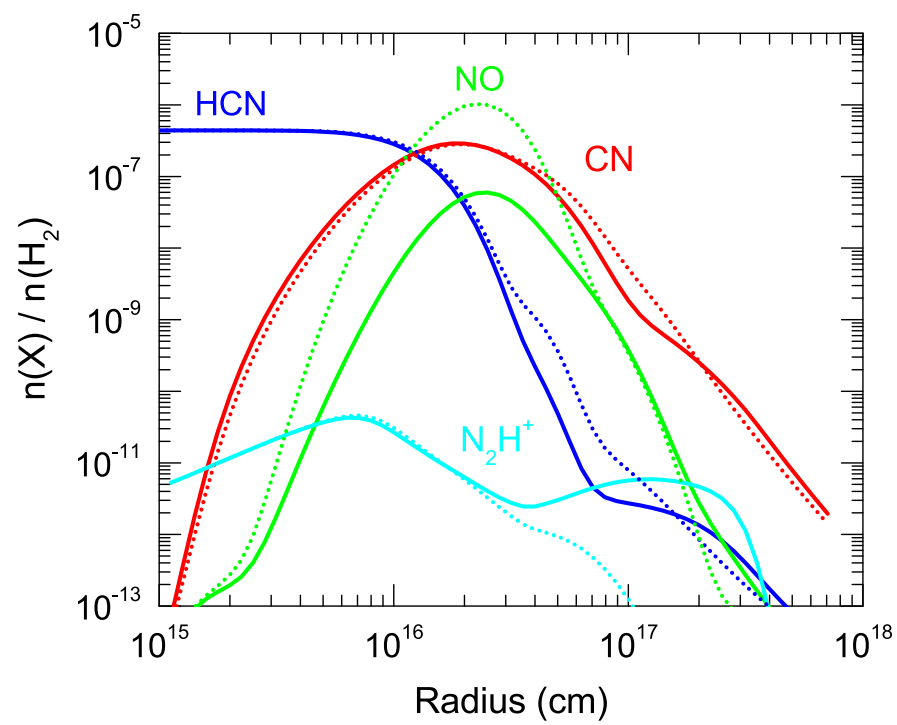

Fig. 6. Fractional abundances of $\mathrm{HCN}, \mathrm{CN}, \mathrm{N}_{2} \mathrm{H}^{+}$, and $\mathrm{NO}$, relative to $\mathrm{H}_{2}$, as a function of radius. Solid and dashed lines exhibit the results calculated from the SS model (Li et al. 2014) and those based on the updated model of McElroy et al. (2013), respectively.

some daughter species whose chemistry is directly related to these species, e.g., $\mathrm{NO}$ and $\mathrm{N}_{2} \mathrm{H}^{+}$, as illustrated in Fig. 6. Other species, for instance $\mathrm{HCN}$ and $\mathrm{CN}$, are less affected.

Another highly abundant species, $\mathrm{H}_{2} \mathrm{O}$, is found to be significantly self-shielded in high abundance regions such as the planet-forming zones of circumstellar disks (Bethell \& Bergin 2009). In the case of O-rich AGB stars, the self-shielding of $\mathrm{H}_{2} \mathrm{O}$ is only considerable at radii less than $2 \times 10^{15} \mathrm{~cm}$, where the photodissociation of $\mathrm{H}_{2} \mathrm{O}$ has not yet started to dominate its chemistry. This implies that the abundance of $\mathrm{H}_{2} \mathrm{O}$ is too low to be self-shielded at larger radii where photodissociation becomes 
significant, and it is not necessary to consider self-shielding of $\mathrm{H}_{2} \mathrm{O}$ in the CSE of AGB stars.

\subsection{O-bearing species}

We now discuss the chemistry of daughter species with column densities higher than $10^{11} \mathrm{~cm}^{-2}$ based on their chemical families. To avoid repetition, species that can be included in multiple subsections are only discussed once. For instance, the molecule $\mathrm{CNO}$, which could be discussed in the subsections of $\mathrm{C}-, \mathrm{O}-$, or $\mathrm{N}$-bearing species, is only discussed in the section for $\mathrm{N}$-bearing species.

The dominant chemistry for many species depends on radius owing to the large gradient in the physical conditions (i.e., visual extinction, temperatures, and gas density) in the CSEs of AGB stars. Thus, the dominating formation and destruction routes might be different in other O-rich AGB stars. In addition, different assumed abundances of parent species may lead to quite different reaction paths for the daughter species.

The most abundant $\mathrm{O}$-bearing daughter species triggered by the chemistry of $\mathrm{H}_{2} \mathrm{O}$ are $\mathrm{OH}, \mathrm{O}_{2}$, and $\mathrm{H}_{3} \mathrm{O}^{+}$, whose radii of peak abundance are found in the region of $(1-4) \times 10^{16} \mathrm{~cm}$, as shown in panel (a) of Fig. B.1. The chemistry of $\mathrm{OH}$ and $\mathrm{O}_{2}$ is relatively simple; they form mainly via the reactions

$\mathrm{H}_{2} \mathrm{O}+\mathrm{h} v \rightarrow \mathrm{OH}+\mathrm{H}$

$\mathrm{OH}+\mathrm{O} \rightarrow \mathrm{O}_{2}+\mathrm{H}$.

$\mathrm{H}_{3} \mathrm{O}^{+}$is mainly formed through

$\mathrm{H}_{2} \mathrm{O}^{+}+\mathrm{H}_{2} \rightarrow \mathrm{H}_{3} \mathrm{O}^{+}+\mathrm{H}$

where $\mathrm{H}_{2} \mathrm{O}^{+}$comes from various routes with the relative importance depending on radius, for instance,

$\mathrm{H}_{3}^{+}+\mathrm{O} \rightarrow \mathrm{H}_{2} \mathrm{O}^{+}+\mathrm{H}$,

$\mathrm{OH}^{+}+\mathrm{H}_{2} \rightarrow \mathrm{H}_{2} \mathrm{O}^{+}+\mathrm{H}$,

$\mathrm{H}_{2} \mathrm{O}+\mathrm{h} v \rightarrow \mathrm{H}_{2} \mathrm{O}^{+}+\mathrm{e}^{-}$.

In the inner CSE, e.g., $\lesssim 2 \times 10^{15} \mathrm{~cm}, \mathrm{OH}^{+}$is mainly generated by

$\mathrm{He}^{+}+\mathrm{H}_{2} \mathrm{O} \rightarrow \mathrm{OH}^{+}+\mathrm{H}+\mathrm{He}$,

where $\mathrm{He}^{+}$is generated by cosmic-ray ionization. Direct $\mathrm{OH}$ photoionization becomes important for radii within $10^{17} \mathrm{~cm}$, while $\mathrm{H}_{3}^{+}+\mathrm{O}$ produces $\mathrm{OH}^{+}$at larger radii. As noted above, this is different from traditional diffuse/PDR chemistry (e.g., Gerin et al. 2010) because of the high abundance of $\mathrm{OH}$ here. $\mathrm{H}_{3}^{+}$is formed by the reaction between $\mathrm{H}_{2}$ and $\mathrm{H}_{2}^{+}$, where $\mathrm{H}_{2}^{+}$comes from the cosmic-ray ionization of $\mathrm{H}_{2}$.

The destruction of most O-bearing species is dominated by photodissociation. In addition to $\mathrm{H}_{2} \mathrm{O}$, photodissociation of other O-bearing species, e.g., $\mathrm{SiO}, \mathrm{SO}, \mathrm{CO}_{2}$, and $\mathrm{PO}$, among others, can liberate atomic oxygen at the outer edge of the CSE. CO is the main contributor at radii greater than $10^{17} \mathrm{~cm}$, where $A_{v}$ is less than $0.3 \mathrm{mag}$. The chemistry of $\mathrm{CO}$ is discussed in the following section. The $\mathrm{O}$-bearing cations are predominantly destroyed by dissociative recombination with electrons; e.g., $\mathrm{H}_{3} \mathrm{O}^{+}$ can be destroyed by the reaction

$\mathrm{H}_{3} \mathrm{O}^{+}+\mathrm{e}^{-} \rightarrow$ products.

The fraction of electrons is an important factor in the ionmolecule chemistry (see panel a of Fig. B.1). Initially, at inner radii, e.g., $\lesssim 2 \times 10^{15} \mathrm{~cm}$, electrons mainly arise from the cosmicray ionization of $\mathrm{H}_{2}$,

$\mathrm{H}_{2}+\mathrm{crp} \rightarrow \mathrm{H}_{2}^{+}+\mathrm{e}^{-}$,

where crp denotes cosmic-ray particles. As the radius increases to the region where the photodissociation of $\mathrm{H}_{2} \mathrm{O}$ becomes important, for instance at $10^{16} \mathrm{~cm}$, electrons are produced by the process shown in Eq. (7). Farther out in the CSE, e.g., $3 \times 10^{16} \mathrm{~cm}$, contributions are mainly from three channels:

$\mathrm{C}+\mathrm{h} v \rightarrow \mathrm{C}^{+}+\mathrm{e}^{-}$

$\mathrm{S}+\mathrm{h} v \rightarrow \mathrm{S}^{+}+\mathrm{e}^{-}$

$\mathrm{Si}+\mathrm{h} v \rightarrow \mathrm{Si}^{+}+\mathrm{e}^{-}$.

At the edge of the CSE, with radius greater than $10^{17} \mathrm{~cm}$, the electron density is controlled by the most abundant element, $\mathrm{C}$. Farther in, the electron fraction as a function of radius is significantly affected by the assumption of key parent species, especially $\mathrm{H}_{2} \mathrm{O}, \mathrm{CO}, \mathrm{SiS}$, and $\mathrm{SiO}$.

\subsection{C-bearing species}

Carbon is mostly in the form of $\mathrm{CO}$ before it is converted into $\mathrm{C}^{+}$at the edge of the envelope (panel b of Fig. B.1). The $\mathrm{CO} \rightarrow$ $\mathrm{C} \rightarrow \mathrm{C}^{+}$conversion controls the carbon chemistry. The non-selfshielding parent species, $\mathrm{CO}_{2}$, is less abundant and photodissociates from a radius of $3 \times 10^{16} \mathrm{~cm}$ outwards. Four carbon compounds, $\mathrm{CH}, \mathrm{CH}_{2}, \mathrm{CH}_{3}^{+}$, and $\mathrm{CH}_{2}^{+}$are found to be quite abundant, and are distributed at radii greater than $3 \times 10^{16} \mathrm{~cm}$ where the temperature is less than $50 \mathrm{~K}$ (in our model $\sim 10 \mathrm{~K}$; see Fig. 2). Therefore, their emission would only be detectable from low rotational energy levels.

The formation of $\mathrm{CH}_{2}$ varies with radius. For instance, at $3 \times 10^{16} \mathrm{~cm}$, it is mainly formed by

$\mathrm{SiCH}_{2}^{+}+\mathrm{e}^{-} \rightarrow \mathrm{Si}+\mathrm{CH}_{2}$.

The chemistry of $\mathrm{SiCH}_{2}^{+}$is discussed in Sect. 3.6. At $10^{17} \mathrm{~cm}$, $\mathrm{CH}_{2}$ is mainly produced by

$\mathrm{CH}_{3}^{+}+\mathrm{e}^{-} \rightarrow \mathrm{CH}_{2}+\mathrm{H}$.

In both cases, $\mathrm{CH}$ is formed through

$\mathrm{CH}_{3}^{+}+\mathrm{e}^{-} \rightarrow \mathrm{CH}+\mathrm{H}_{2}$,

whereas $\mathrm{CH}_{3}^{+}$arises from the following process at all radii:

$\mathrm{CH}_{2}^{+}+\mathrm{H}_{2} \rightarrow \mathrm{CH}_{3}^{+}+\mathrm{H}$.

$\mathrm{CH}_{2}^{+}$is generated through multiple channels, by

$\mathrm{C}^{+}+\mathrm{H}_{2} \rightarrow \mathrm{CH}_{2}^{+}+\mathrm{h} v$,

$\mathrm{CH}^{+}+\mathrm{H}_{2} \rightarrow \mathrm{CH}_{2}^{+}+\mathrm{H}$,

and

$\mathrm{CH}_{2}+\mathrm{h} v \rightarrow \mathrm{CH}_{2}^{+}+\mathrm{e}^{-}$.

In the above reactions, most of the $\mathrm{CH}^{+}$is produced via the photoionization of $\mathrm{CH}$, and is destroyed by the process described in Eq. (19) at all radii. The destruction of the neutral carbon hydrides, $\mathrm{CH}$ and $\mathrm{CH}_{2}$, is dominated by photodissociation, whereas that of most cations, e.g., $\mathrm{CH}_{3}^{+}$, is via dissociative recombination. Interestingly, the destruction of $\mathrm{CH}_{2}^{+}$is dominated by Eq. (17) rather than electrons. The chemistry of four other C-bearing species, namely $\mathrm{C}_{2}, \mathrm{C}_{2} \mathrm{H}, \mathrm{CH}_{3} \mathrm{OH}$, and $\mathrm{HCO}^{+}$, are discussed in Sect. 3.12. 


\subsection{N-bearing species}

The chemistry of the parent species ammonia, $\mathrm{NH}_{3}$, controls the distribution of most $\mathrm{N}$-bearing species, among which eight are found above the $10^{-11}$ abundance threshold (panel $\mathrm{c}$ of Fig. B.1). These species are mainly located at radii between $5 \times 10^{15}$ and $5 \times 10^{16} \mathrm{~cm}$ from the star. The most abundant daughter species generated by $\mathrm{NH}_{3}$ are $\mathrm{NH}_{2}$ and $\mathrm{NH}$, which have peak fractional abundances around $10^{-6}$. By analogy with the corresponding oxygen and carbon species, $\mathrm{NH}_{2}$ is produced by the photodissociation of $\mathrm{NH}_{3}$ at radii less than its peak radius, $2.2 \times 10^{16} \mathrm{~cm}$, whereas dissociative recombination of $\mathrm{NH}_{3}^{+}$with $\mathrm{e}^{-}$dominates at greater radii. $\mathrm{NH}$ is the product of the photodissociation of $\mathrm{NH}_{3}$ and $\mathrm{NH}_{2}$ throughout the entire CSE, but with a significant contribution from the reaction between $\mathrm{NH}_{3}^{+}$and $\mathrm{e}^{-}$when the radius is greater than $1 \times 10^{17} \mathrm{~cm}$.

The relatively abundant cation, $\mathrm{NH}_{3}^{+}$, is mainly formed through the photoionization of $\mathrm{NH}_{3}$ for radii inside its peak abundance, and by the reaction between $\mathrm{NH}_{2}^{+}$and $\mathrm{H}_{2}$ at greater radii. $\mathrm{NH}_{2}^{+}$comes from the photoionization of $\mathrm{NH}_{2}$ and reactions between $\mathrm{NH}^{+}$and $\mathrm{H}_{2}$. The chemistry of $\mathrm{NH}_{4}^{+}$is less complex, and forms mainly via the reaction between $\mathrm{NH}_{3}^{+}$and $\mathrm{H}_{2}$. Only at very inner radii, e.g., $2 \times 10^{15} \mathrm{~cm}$, is it formed by the reaction of $\mathrm{NH}_{3}$ and $\mathrm{H}_{3} \mathrm{O}^{+}$.

The chemistry of $\mathrm{NH}_{3}$ also gives rise to other $\mathrm{N}$-bearing species, such as $\mathrm{HNO}, \mathrm{NO}, \mathrm{HNC}$, and $\mathrm{HCNH}^{+}$(panel $\mathrm{d}$ of Fig. B.1) at radii between $10^{16}-10^{17} \mathrm{~cm}$. First, the breaking down of $\mathrm{NH}_{3}$ generates $\mathrm{NH}_{2}$, which reacts with atomic $\mathrm{O}$ and produces HNO. Then, HNO is photodissociated to NO. The formation channels of NO vary with radius, e.g., a non-negligible amount of $\mathrm{NO}$ is formed from the reaction between $\mathrm{N}$ and $\mathrm{OH}$ in the inner regions $\leq 2 \times 10^{15} \mathrm{~cm}$, and by $\mathrm{OCN}+\mathrm{O}$ at radii greater than $5 \times 10^{17} \mathrm{~cm}$.

Throughout the CSE, HNC is mainly produced via $\mathrm{HCNH}^{+}$, in the following two reactions

$\mathrm{HCNH}^{+}+\mathrm{NH}_{3} \rightarrow \mathrm{HNC}+\mathrm{NH}_{4}^{+}$,

and

$\mathrm{HCNH}^{+}+\mathrm{e}^{-} \rightarrow \mathrm{HNC}+\mathrm{H}$

The formation of $\mathrm{HCNH}^{+}$is complex. At radii less than $10^{16} \mathrm{~cm}$ it is mainly formed via the reaction between $\mathrm{H}_{3} \mathrm{O}^{+}$and $\mathrm{HCN}$. However, at larger radii it is entirely formed via

$\mathrm{HCN}^{+}+\mathrm{H}_{2} \rightarrow \mathrm{HCNH}^{+}+\mathrm{H}$

where $\mathrm{HCN}^{+}$is the product of reactions between $\mathrm{C}^{+}+\mathrm{NH}_{2}$, and $\mathrm{CN}^{+}+\mathrm{H}_{2} \cdot \mathrm{CN}^{+}$comes from the reaction between $\mathrm{C}^{+}$and $\mathrm{NH}$. Therefore, $\mathrm{HCN}$ contributes most to the formation of $\mathrm{HNC}$ in the inner regions whereas $\mathrm{NH}_{3}$ plays the most important role in the outer regions.

Another important N-bearing parent species, $\mathrm{HCN}$, controls the chemistry of $\mathrm{CN}, \mathrm{OCN}$, and $\mathrm{NH}_{2} \mathrm{CN}$ (panel e of Fig. B.1). In our model, $\mathrm{HCN}$ starts to photodissociate at a radius of $10^{16} \mathrm{~cm}$, breaking down into its daughter species, $\mathrm{CN}$. We note that there are other (but very minor) routes leading to the formation of $\mathrm{CN}$ at its peak radius, for instance, the photodissociation of HNC, and the reaction between OCN and $\mathrm{H}$. Its destruction at large radii is simply dominated by photodissociation.

The species OCN is mainly formed via

$\mathrm{OH}+\mathrm{CN} \rightarrow \mathrm{OCN}+\mathrm{H}$ at radii less than $8 \times 10^{16} \mathrm{~cm}$, whereas dissociative recombination dominates its formation at greater radii, via

$\mathrm{HNCO}^{+}+\mathrm{e}^{-} \rightarrow \mathrm{OCN}+\mathrm{H}$, and

$\mathrm{HOCN}^{+}+\mathrm{e}^{-} \rightarrow \mathrm{OCN}+\mathrm{H}$

with reaction rate constants that are assumed equal, $1.5 \times$ $10^{-7} \mathrm{~cm}^{3} \mathrm{~s}^{-1}$. However, $\mathrm{HNCO}^{+}$and $\mathrm{HOCN}^{+}$are totally formed by the reaction between $\mathrm{OCN}$ and $\mathrm{H}_{3}^{+}$(with a reaction rate constant of $1.6 \times 10^{-8} \mathrm{~cm}^{3} \mathrm{~s}^{-1}$ ) and so comprise a cyclic formation pathway and not the dominant route to OCN bond formation. The rates for the above reactions are adopted from Quan et al. (2010), where more detailed discussions on these species can be found.

The chemistry of $\mathrm{NH}_{2} \mathrm{CN}$ is dependent on both $\mathrm{NH}_{3}$ and $\mathrm{HCN}$. This species is simply formed by the reaction

$\mathrm{NH}_{3}+\mathrm{CN} \rightarrow \mathrm{NH}_{2} \mathrm{CN}+\mathrm{H}$

and destroyed by photodissociation,

$\mathrm{NH}_{2} \mathrm{CN}+\mathrm{h} v \rightarrow \mathrm{NH}_{2}+\mathrm{CN}$.

We note that more recent rate coefficients and branching ratios for the $\mathrm{CH}+\mathrm{NO}$ reaction can be found in Bergeat et al. (1999) and references therein. These make no significant differences to our results.

\subsection{Si-bearing species}

The photodissociation/photoionization processes of parent species, $\mathrm{SiO} / \mathrm{SiS} \rightarrow \mathrm{Si} \rightarrow \mathrm{Si}^{+}$, control the silicon chemistry and gives rise to other Si-bearing species (panels $g$ and $h$ of Fig. B.1). Similar to N-bearing species, most silicon compounds have peak abundances for radii between $10^{16}$ and $10^{17} \mathrm{~cm}$. In total, seven high-abundance daughter molecules are found: $\mathrm{SiS}^{+}$, $\mathrm{SiO}_{2}, \mathrm{SiOH}^{+}, \mathrm{SiN}, \mathrm{HNSi}, \mathrm{SiN}^{+}$and $\mathrm{SiNH}_{2}^{+}$.

In the inner layers, at radii smaller than $2 \times 10^{16} \mathrm{~cm}$, the abundance of $\mathrm{Si}$ is higher than $\mathrm{Si}^{+}$and its formation is dominated by the photodissociation of $\mathrm{SiO}$. As the distance from the star increases, $\mathrm{Si}$ is photoionized to form $\mathrm{Si}^{+}$and therefore contributes to the electron density. $\mathrm{SiS}^{+}$comes from $\mathrm{SiS}$, via charge exchange,

$\mathrm{SiS}+\mathrm{S}^{+} \rightarrow \mathrm{SiS}^{+}+\mathrm{S}$

around its peak radius, and by the reaction between $\mathrm{C}^{+}$and $\mathrm{SiS}$ in other regions. Its destruction is dominated by

$\mathrm{SiS}^{+}+\mathrm{e}^{-} \rightarrow \mathrm{Si}+\mathrm{S}$.

The presence of $\mathrm{OH}$ at a high abundance $\left(\sim 10^{-5}\right)$ generates a significant abundance of $\mathrm{SiOH}^{+}$in an O-rich CSE,

$\mathrm{Si}^{+}+\mathrm{OH} \rightarrow \mathrm{SiO}^{+}+\mathrm{H}$

$\mathrm{SiO}^{+}+\mathrm{H}_{2} \rightarrow \mathrm{SiOH}^{+}$

which is destroyed by dissociative recombination in the outer layers,

$\mathrm{SiOH}^{+}+\mathrm{e}^{-} \rightarrow \mathrm{Si}+\mathrm{OH}$

$\mathrm{SiOH}^{+}+\mathrm{e}^{-} \rightarrow \mathrm{SiO}+\mathrm{H}$.

Reactions (31) and (32) dominate the formation and destruction of $\mathrm{SiO}^{+}$. Similarly, the parent species $\mathrm{NH}_{3}$ generates $\mathrm{NH}$, then 
increases the abundance of $\mathrm{SiN}^{+}$, which is formed and destroyed via the following processes at all radii:

$\mathrm{NH}+\mathrm{Si}^{+} \rightarrow \mathrm{SiN}^{+}+\mathrm{H}$,

$\mathrm{SiN}^{+}+\mathrm{e}^{-} \rightarrow \mathrm{Si}+\mathrm{N}$.

$\mathrm{SiNH}_{2}^{+}$is formed and destroyed in a very similar way, via

$$
\begin{aligned}
& \mathrm{NH}_{3}+\mathrm{Si}^{+} \rightarrow \mathrm{SiNH}_{2}^{+}+\mathrm{H}, \\
& \mathrm{SiNH}_{2}^{+}+\mathrm{e}^{-} \rightarrow \mathrm{SiN}+\mathrm{H}_{2}, \\
& \mathrm{SiNH}_{2}^{+}+\mathrm{e}^{-} \rightarrow \mathrm{HNSi}+\mathrm{H} .
\end{aligned}
$$

The chemistry of $\mathrm{SiO}_{2}$ is triggered by $\mathrm{SiO}$, via the following reaction throughout the envelope:

$\mathrm{SiO}+\mathrm{OH} \rightarrow \mathrm{SiO}_{2}+\mathrm{H}$.

$\mathrm{SiN}$ is mainly generated by Eq. (38) within $10^{16} \mathrm{~cm}$, but from the photodissociation of $\mathrm{HNSi}$ at greater radii. At all radii, HNSi is produced by Eq. (39). The destruction of all of these neutral silicon compounds is dominated by photodissociation, although their actual rates are quite uncertain and are experimentally difficult to measure.

The species HCSi peaks at a relatively larger radius, $4 \times$ $10^{16} \mathrm{~cm}$, arising from $\mathrm{SiS}$ through

$\mathrm{SiS}+\mathrm{C}^{+} \rightarrow \mathrm{SiC}^{+}+\mathrm{S}$

$\mathrm{SiC}^{+}+\mathrm{H}_{2} \rightarrow \mathrm{HCSi}^{+}+\mathrm{H}$,

$\mathrm{HCSi}^{+}+\mathrm{H}_{2} \rightarrow \mathrm{SiCH}_{2}^{+}+\mathrm{H}$,

and

$\mathrm{SiCH}_{2}^{+}+\mathrm{e}^{-} \rightarrow \mathrm{HCSi}+\mathrm{H}$,

and is dissociated by UV photons.

\subsection{S-bearing species}

The conversion from the parents $\mathrm{SO}_{2} / \mathrm{SO} / \mathrm{SiS} \rightarrow \mathrm{S} \rightarrow \mathrm{S}^{+}$in the CSE is the key to the sulphur chemistry (panels $i$ and $j$ of Fig. B.1). The increase in atomic $S$ is caused by the photodissociation of $\mathrm{SO}$ and $\mathrm{SO}_{2}$ (first releasing $\mathrm{SO}$ then $\mathrm{S}$ ) at radii less than $10^{16} \mathrm{~cm}$, with a contribution from the breaking down of SiS at larger radii, $\geq 3 \times 10^{16} \mathrm{~cm}$. Beyond this point, $\mathrm{S}$ is converted into $\mathrm{S}^{+}$by photoionization. The less abundant parent species, $\mathrm{H}_{2} \mathrm{~S}$ and HS, play a less important role in the chemical network.

In total, we find four abundant daughter species. At all radii, OCS is formed via

$\mathrm{CS}+\mathrm{OH} \rightarrow \mathrm{OCS}+\mathrm{H}$,

and NS is formed via

$\mathrm{NH}+\mathrm{S} \rightarrow \mathrm{NS}+\mathrm{H}$.

Similar to other neutral species, the destruction of OCS and NS is dominated by photodissociation. $\mathrm{NS}^{+}$has a relatively low abundance, coming from $\mathrm{NH}$ reacting with $\mathrm{S}^{+}$. Like most cations, it is destroyed by dissociative recombination. $\mathrm{SO}^{+}$is produced by the following two reactions,

$\mathrm{SO}+\mathrm{h} v \rightarrow \mathrm{SO}^{+}+\mathrm{e}^{-}$,

$\mathrm{OH}+\mathrm{S}^{+} \rightarrow \mathrm{SO}^{+}+\mathrm{H}$,

and, again, is destroyed by dissociative recombination.

\subsection{P-bearing species}

$\mathrm{PN}$ and $\mathrm{PO}$ are the two phosphorus-bearing parent species in our model. The conversion process $\mathrm{PN} / \mathrm{PO} \rightarrow \mathrm{P} \rightarrow \mathrm{P}^{+}$, which is dominated by the photodissociation and photoionization, controls the phosphorus chemistry (panel k in Fig. B.1).

There are no experimental or theoretical studies of PN photodissociation. When employing a lower photodissociation rate of $5 \times 10^{-12} \mathrm{~s}^{-1}$ provided by the RATE12 reaction network, our model produces a significant and likely unrealistic abundance of $\mathrm{PN}$ at very large radii where all other molecular species have been destroyed by the interstellar radiation field. This is also due to the neglect of PN photoionization in RATE12 despite the low ionization threshold of this molecule (Wu \& Fehlner 1975) and a photoabsorption cross section that is likely to be comparable to that of electronically similar $\mathrm{CO}$ and $\mathrm{N}_{2}$. Thus, we adopted an improved PN photodissociation rate of $2.0 \times 10^{-10} \mathrm{~s}^{-1}$, which is comparable to the $\mathrm{N}_{2}$ photodissociation rate ( $\mathrm{Li}$ et al. 2013), together with an additional photoionization rate of $2.0 \times 10^{-10} \mathrm{~s}^{-1}$ with $\gamma=3.0$ for

$\mathrm{PN}+\mathrm{h} v \rightarrow \mathrm{PN}^{+}+\mathrm{e}^{-}$

to update the phosphorus chemistry.

Three species, $\mathrm{HPN}^{+}, \mathrm{PH}$, and $\mathrm{PN}^{+}$, are found to be abundant. $\mathrm{HPN}^{+}$is mainly formed via

$\mathrm{H}_{3} \mathrm{O}^{+}+\mathrm{PN} \rightarrow \mathrm{HPN}^{+}+\mathrm{H}_{2} \mathrm{O}$

and destroyed by three channels assumed to have equal branching ratios:

$\mathrm{HPN}^{+}+\mathrm{e}^{-} \rightarrow \mathrm{PH}+\mathrm{N}$,

$\mathrm{HPN}^{+}+\mathrm{e}^{-} \rightarrow \mathrm{P}+\mathrm{NH}$

$\mathrm{HPN}^{+}+\mathrm{e}^{-} \rightarrow \mathrm{PN}+\mathrm{H}$.

$\mathrm{PH}$ arises from Eq. (51) at radii smaller than $1 \times 10^{16} \mathrm{~cm}$. Contribution from the routes

$\mathrm{H}_{2} \mathrm{O}+\mathrm{P}^{+} \rightarrow \mathrm{HPO}^{+}+\mathrm{H}$,

$\mathrm{HPO}^{+}+\mathrm{e}^{-} \rightarrow \mathrm{PH}+\mathrm{O}$

becomes more important as the radius increases. The peak abundance of $\mathrm{PH}$ varies with the abundance of $\mathrm{P}^{+}$. The destruction of the neutral species $\mathrm{PH}$ is dominated by photodissociation. The main change of employing the modified PN photodissociation and photoionization rates is the destruction of the unrealistically high density of $\mathrm{PN}$ at large radii, and a resultant increase in $\mathrm{PN}^{+}$ abundance. The main destruction pathway of $\mathrm{PN}^{+}$is dissociative recombination.

Further work on the PN photoprocesses is necessary to constrain our estimated rates.

\subsection{Cl-bearing species}

The chemistry of Cl-bearing species is initiated by the photodissociation of the parent species, $\mathrm{HCl}$ (panel $\mathrm{f}$ of Fig. B.1). The most abundant Cl-bearing daughter molecule in this model is $\mathrm{H}_{2} \mathrm{Cl}^{+}$. The breakdown of $\mathrm{HCl}$ leads to two species,

$\mathrm{HCl}+\mathrm{h} v \rightarrow \mathrm{Cl}+\mathrm{H}$,

$\mathrm{HCl}+\mathrm{h} v \rightarrow \mathrm{HCl}^{+}+\mathrm{e}^{-}$.

At all radii, $\sim 90 \%$ of $\mathrm{HCl}$ goes to Eq. (56) whereas $\sim 10 \%$ goes to Eq. (57). $\mathrm{H}_{2} \mathrm{Cl}^{+}$is formed via

$\mathrm{HCl}^{+}+\mathrm{H}_{2} \rightarrow \mathrm{H}_{2} \mathrm{Cl}^{+}+\mathrm{H}$ 
and destroyed by

$\mathrm{H}_{2} \mathrm{Cl}^{+}+\mathrm{e}^{-} \rightarrow \mathrm{Cl}+\mathrm{H}_{2}$.

The cation $\mathrm{Cl}^{+}$arises from the photoionization of $\mathrm{Cl}$. It is worth mentioning that $\mathrm{H}_{2} \mathrm{Cl}^{+}, \mathrm{HCl}^{+}$, and $\mathrm{H}_{2} \mathrm{O}^{+}$have been detected by the Herschel space observatory in the diffuse interstellar medium (e.g. Gerin et al. 2010, 2013; Lis et al. 2010; De Luca et al. 2012; Neufeld et al. 2012). Another Cl-bearing species, $\mathrm{NaCl}$, has been found in IK Tau with an abundance of $4 \times 10^{-9}$ (Milam et al. 2007). Unfortunately we do not have the chemistry of Na-bearing species in our network, thereby an extension of the chemical network would be useful for a further understanding of the chlorine chemistry. Our model shows that the abundance of $\mathrm{CCl}^{+}$is negligible and is not shown in the plot.

\subsection{F-bearing species}

This is the first time that F-chemistry is included in a CSE model of an O-rich AGB star. The photodissociation of the parent species, HF, liberates atomic $\mathrm{F}$ and gives rise to other F-bearing species (panel 1 of Fig. B.1). Three daughter species, $\mathrm{SiF}^{+}, \mathrm{CF}^{+}$, and $\mathrm{H}_{2} \mathrm{~F}^{+}$are found to be very abundant. In particular, $\mathrm{SiF}^{+}$has not been detected in the ISM because of the depleted elemental abundance of silicon. However, we predict that it may be detectable in IK Tau at a radius of $\sim 10^{16} \mathrm{~cm}$. At all radii, $\mathrm{SiF}^{+}$and $\mathrm{CF}^{+}$are formed by the reactions

$\mathrm{HF}+\mathrm{Si}^{+} \rightarrow \mathrm{SiF}^{+}+\mathrm{H}$

$\mathrm{HF}+\mathrm{C}^{+} \rightarrow \mathrm{CF}^{+}+\mathrm{H}$,

and are destroyed by

$\mathrm{SiF}^{+}+\mathrm{e}^{-} \rightarrow \mathrm{Si}+\mathrm{F}$,

$\mathrm{CF}^{+}+\mathrm{e}^{-} \rightarrow \mathrm{C}+\mathrm{F}$.

$\mathrm{H}_{2} \mathrm{~F}^{+}$is formed via two routes,

$\mathrm{HF}+\mathrm{H}_{3}^{+} \rightarrow \mathrm{H}_{2} \mathrm{~F}^{+}+\mathrm{H}_{2}$,

$\mathrm{HF}^{+}+\mathrm{H}_{2} \rightarrow \mathrm{H}_{2} \mathrm{~F}^{+}+\mathrm{H}$,

where $\mathrm{HF}^{+}$arises from

$\mathrm{He}^{+}+\mathrm{HF} \rightarrow \mathrm{F}^{+}+\mathrm{He}+\mathrm{H}$,

and

$\mathrm{F}^{+}+\mathrm{H}_{2} \rightarrow \mathrm{HF}^{+}+\mathrm{H}$.

The destruction of $\mathrm{H}_{2} \mathrm{~F}^{+}$is dominated by

$\mathrm{H}_{2} \mathrm{~F}^{+}+\mathrm{e}^{-} \rightarrow \mathrm{F}+\mathrm{H}_{2}$

and

$\mathrm{H}_{2} \mathrm{~F}^{+}+\mathrm{e}^{-} \rightarrow \mathrm{HF}+\mathrm{H}$

\subsection{Comparison with observations}

The calculated fractional abundances, peak radii, and total column densities of the top 37 daughter species in IK Tau are presented in Table 3. Observational information is only available for $\mathrm{CN}$ and $\mathrm{HCO}^{+}$. For $\mathrm{CN}$ the calculated peak abundance, total column density, and peak radius are in good agreement with the observations from Kim et al. (2010) and Decin et al. (2010b). For $\mathrm{HCO}^{+}$the calculated peak radius is in excellent agreement with
Table 3. Calculated total column densities, peak abundances (relative to $\mathrm{H}_{2}$ ), and peak radii of the daughter molecules in the CSE of the O-rich AGB star, IK Tau.

\begin{tabular}{|c|c|c|c|c|}
\hline No. & Species & $\begin{array}{c}N_{\text {total }} \\
\left(\mathrm{cm}^{-2}\right)\end{array}$ & Peak abundance & $\begin{array}{c}\text { Peak radius } \\
(\mathrm{cm})\end{array}$ \\
\hline 1 & $\mathrm{OH}$ & $5.4(15)$ & $2.8(-5)$ & $1.8(16)$ \\
\hline 2 & $\mathrm{C}$ & $4.0(14)$ & $9.9(-6)$ & $1.6(17)$ \\
\hline 3 & $\mathrm{NH}_{2}$ & $1.4(14)$ & $6.2(-7)$ & $1.4(16)$ \\
\hline 4 & $\mathrm{NH}^{2}$ & $8.2(13)$ & $4.8(-7)$ & $1.8(16)$ \\
\hline 5 & $\mathrm{O}_{2}$ & $7.8(13)$ & $6.5(-7)$ & $2.0(16)$ \\
\hline 6 & $\mathrm{CN}$ & $5.8(13)$ & $2.9(-7)^{a}$ & $1.8(16)$ \\
\hline 7 & $\mathrm{SiOH}^{+}$ & $4.0(13)$ & $8.1(-8)$ & $1.3(16)$ \\
\hline 8 & $\mathrm{SiN}$ & $1.3(13)$ & $9.1(-8)$ & $1.8(16)$ \\
\hline 9 & HNSi & $1.2(13)$ & $8.2(-8)$ & $1.6(16)$ \\
\hline 10 & $\mathrm{HNO}$ & $7.9(12)$ & $6.1(-8)$ & $2.2(16)$ \\
\hline 11 & $\mathrm{SO}^{+}$ & $6.6(12)$ & $1.5(-8)$ & $6.3(15)$ \\
\hline 12 & NO & $5.9(12)$ & $6.0(-8)$ & $2.5(16)$ \\
\hline 13 & $\mathrm{SiO}_{2}$ & $5.9(12)$ & $2.4(-8)$ & $1.6(16)$ \\
\hline 14 & $\mathrm{NH}_{4}^{+}$ & $3.7(12)$ & 4.1(-9) & $4.0(15)$ \\
\hline 15 & $\mathrm{SiC}^{-}$ & $3.3(12)$ & $6.7(-8)$ & $4.5(16)$ \\
\hline 16 & $\mathrm{H}_{3} \mathrm{O}^{+}$ & $3.0(12)$ & $7.1(-9)$ & 7.1(15) \\
\hline 17 & OCN & $2.7(12)$ & $1.9(-8)$ & $2.5(16)$ \\
\hline 18 & $\mathrm{SiS}^{+}$ & $2.4(12)$ & $1.2(-8)$ & $2.5(16)$ \\
\hline 19 & $\mathrm{CH}$ & $2.0(12)$ & $2.6(-8)$ & $4.5(16)$ \\
\hline 20 & $\mathrm{CH}_{2}$ & $1.2(12)$ & $2.1(-8)$ & $4.0(16)$ \\
\hline 21 & $\mathrm{HCSi}$ & $1.1(12)$ & $2.2(-8)$ & $4.0(16)$ \\
\hline 22 & OCS & $1.1(12)$ & $3.6(-9)$ & $8.9(15)$ \\
\hline 23 & NS & $9.1(11)$ & 7.6(-9) & $2.5(16)$ \\
\hline 24 & $\mathrm{NH}_{3}^{+}$ & $8.9(11)$ & $2.8(-9)$ & $7.9(15)$ \\
\hline 25 & $\mathrm{SiNH}_{2}^{+}$ & $6.5(11)$ & $2.6(-9)$ & $7.9(15)$ \\
\hline 26 & $\mathrm{HNC}$ & $3.9(11)$ & $8.9(-10)$ & $8.9(15)$ \\
\hline 27 & $\mathrm{SiF}^{+}$ & $3.3(11)$ & $1.5(-9)$ & $8.9(15)$ \\
\hline 28 & $\mathrm{SiN}^{+}$ & $3.2(11)$ & $2.2(-9)$ & $1.8(16)$ \\
\hline 29 & $\mathrm{PH}$ & $8.8(11)$ & $8.1(-9)$ & $2.5(16)$ \\
\hline 30 & $\mathrm{H}_{3}^{+}$ & $2.5(11)$ & $2.5(-8)$ & 7.1(17) \\
\hline 31 & $\mathrm{SiO}^{+}$ & $2.5(11)$ & $2.7(-9)$ & $2.5(16)$ \\
\hline 32 & $\mathrm{C}_{2}$ & 2.3(11) & 7.6(-9) & $6.3(16)$ \\
\hline 33 & $\mathrm{NH}_{2} \mathrm{CN}$ & $1.9(11)$ & $9.5(-10)$ & $1.3(16)$ \\
\hline 34 & $\mathrm{HCNH}^{+}$ & $1.8(11)$ & $2.0(-10)$ & $5.0(15)$ \\
\hline 35 & $\mathrm{H}_{2} \mathrm{Cl}^{+}$ & $1.7(11)$ & $1.1(-8)$ & 7.1(17) \\
\hline 36 & $\mathrm{HCO}^{+}$ & $1.6(11)^{b}$ & $4.0(-10)^{c}$ & $2.5(16)^{c}$ \\
\hline 37 & $\mathrm{PN}^{+}$ & $3.3(11)$ & $8.2(-10)$ & $6.3(15)$ \\
\hline
\end{tabular}

Notes. $a(b)=a \times 10^{b} .^{(a)}$ Observationally, Decin et al. (2010b) obtained $2(-10)-6(-8)$, whereas Kim et al. (2010) deduced two values, $5.1(-8)$ or $1.6(-7)$, corresponding to two cases where two different radii of $\mathrm{CN}$ were assumed in their models. ${ }^{(b)}$ Observationally, Edwards et al. deduced $1.0(13) \mathrm{cm}^{-2}$ (priv. comm.) ${ }^{(c)}$ Observationally, Pulliam et al. (2011) obtained a peak abundance of $4.4(-8)$ at radius $3.9(16) \mathrm{cm}$.

the observations of Pulliam et al. (2011), but the fractional abundance and total column density are orders of magnitude lower than the observations. This is discussed in the following section. The calculated total column densities of a few parent species, such as $\mathrm{HCN}, \mathrm{CS}, \mathrm{SiS}, \mathrm{SiO}, \mathrm{SO}$, and $\mathrm{SO}_{2}$, are also compared to the observational results (Kim et al. 2010). Agreements are within a factor of 10 demonstrating that the parent species' abundances are not altered significantly by gas-phase chemistry en route through the inner region of the outer envelope. However, we note that we have neglected gas-phase chemical processing through the intermediate envelope which may also affect the parent species' abundances. A thorough comparison with observations requires calculations of the excitation and radiative transfer to compute line intensities rather than column densities, which is beyond the scope of the present work. 


\subsection{Sensitivities to the initial abundances}

The assumed abundance of parent species is of particular importance for the predicted distribution and abundance of daughter species. The identification and abundance of most of these species at inner radii can be well constrained by both observations and theoretical models. However, the initial abundances of some parent species (e.g., $\mathrm{N}_{2}$ ) remain unclear. In addition, there is some discussion in the literature concerning the presence of additional species (e.g., atomic $\mathrm{S}$ and $\mathrm{CH}_{4}$ ) in the inner envelope with non-negligible abundances (see, e.g., Willacy \& Millar 1997; Cherchneff 2006). A possible solution for inferring the presence and abundance of these species is via a few relevant daughter species.

Methane $\left(\mathrm{CH}_{4}\right)$ is not assumed to be a parent species in the current study, based on the conclusions from the observational work of Marvel (2005), where the upper limits on abundances for $\mathrm{C}_{2} \mathrm{H}$ and $\mathrm{CH}_{3} \mathrm{OH}$ (methanol) were deduced to be $9.7 \times 10^{-9}$ and $4.5 \times 10^{-9}$, respectively. Gobrecht et al. (2016) also find that $\mathrm{CH}_{4}$ is not formed efficiently via gas-phase chemistry in the inner wind $\left(r \lesssim 10 R_{*}\right)$ in their model of IK Tau. Our model results are in general agreement with these conclusions. In the earlier photochemical model of Willacy \& Millar (1997), $\mathrm{CH}_{4}$ was assumed to be a parent molecule with a large fractional abundance, $3.0 \times 10^{-5}$, to explain the chemistry of a few carbon species, especially the unexpected presence of $\mathrm{HCN}$ in O-rich envelopes. At that time $\mathrm{HCN}$ was thought to be a daughter species that formed via reactions between atomic $\mathrm{N}, \mathrm{CH}_{2}$, and $\mathrm{CH}_{3}$, which are photoproducts of $\mathrm{N}_{2}$ and $\mathrm{CH}_{4}$. The presence of $\mathrm{CH}_{4}$ as a parent also significantly increases the fractions of both $\mathrm{C}_{2} \mathrm{H}$ and $\mathrm{CH}_{3} \mathrm{OH}$, by which one can constrain the upper limits for methane.

We find that the maximum abundance of $\mathrm{CH}_{4}$ in the $\mathrm{O}$-rich AGB star IK Tau is $2.5 \times 10^{-6}$ in order to be consistent with the observed upper limit for the $\mathrm{C}_{2} \mathrm{H}$ abundance (see panel a of Fig. B.2). Regardless of the inclusion or exclusion $\mathrm{CH}_{4}$ as a parent molecule in the model, the predicted $\mathrm{CH}_{3} \mathrm{OH}$ is at least 5 orders of magnitude lower than its observational upper limit, peaking at radii between $10^{15}-10^{16} \mathrm{~cm}$. One of the most important channels for $\mathrm{CH}_{3} \mathrm{OH}$ formation is via

$\mathrm{CH}_{3}^{+}+\mathrm{H}_{2} \mathrm{O} \rightarrow \mathrm{CH}_{3} \mathrm{OH}_{2}^{+}+\mathrm{h} v$,

$\mathrm{CH}_{3} \mathrm{OH}_{2}^{+}+\mathrm{e}^{-} \rightarrow \mathrm{CH}_{3} \mathrm{OH}+\mathrm{H}$;

the chemistry of $\mathrm{CH}_{3}^{+}$is discussed in Sect. 3.4. We note that in dense interstellar clouds, $\mathrm{CH}_{3} \mathrm{OH}$ is mainly formed on grain surfaces (Watanabe \& Kouchi 2002; Garrod \& Herbst 2006; Geppert et al. 2006; Fuchs et al. 2009) which is neglected here; however, the physical conditions in the CSE are not amenable to ice formation. Typically, ices exist in regions where $A_{v}>1$ which corresponds to the high temperature region $(>600 \mathrm{~K})$ in our model of IK Tau (see Fig. 2). Nevertheless, our results for gas-phase methanol are well within the observed upper limit from Marvel (2005).

At the radii where $\mathrm{C}_{2}$ possesses its peak abundance, it mainly arises from

$\mathrm{C}^{+}+\mathrm{SiC} \rightarrow \mathrm{Si}^{+}+\mathrm{C}_{2}$.

$\mathrm{C}_{2} \mathrm{H}$ can be formed via multiple channels, among which the following routes contribute most:

$\mathrm{C}_{2}+\mathrm{h} v \rightarrow \mathrm{C}_{2}^{+}+\mathrm{e}^{-}$,

$\mathrm{C}_{2}^{+}+\mathrm{H}_{2} \rightarrow \mathrm{C}_{2} \mathrm{H}^{+}+\mathrm{H}$,

$\mathrm{C}_{2} \mathrm{H}^{+}+\mathrm{H}_{2} \rightarrow \mathrm{C}_{2} \mathrm{H}_{2}^{+}+\mathrm{H}$,

$\mathrm{C}_{2} \mathrm{H}_{2}^{+}+\mathrm{e}^{-} \rightarrow \mathrm{C}_{2} \mathrm{H}+\mathrm{H}$.
$\mathrm{HCO}^{+}$has been detected in IK Tau, with an abundance of $4.4 \times 10^{-8}$ at a radius of $3.9 \times 10^{16} \mathrm{~cm}$ (Pulliam et al. 2011). The current model gives a peak radius that is in excellent agreement with this observation, but produces a lower abundance of $\mathrm{HCO}^{+}$. This is the case even where the cosmic-ray ionization rate of $\mathrm{H}_{2}$ is increased with respect to its standard value in our models of $1.2 \times 10^{17} \mathrm{~s}^{-1}$ by a scaling factor $\chi$ up to a value of $\times 1000$. The inclusion of the maximum amount of $\mathrm{CH}_{4}$ consistent with observations, $2.5 \times 10^{-6}$, only increases $\mathrm{HCO}^{+}$by a minor amount (see panel b of Fig. B.2). Another model assuming a higher abundance for the initial $\mathrm{CO}$ only slightly enhances the peak abundance of $\mathrm{HCO}^{+}$. At the radius of its peak abundance, i.e., $3 \times 10^{16} \mathrm{~cm}, \mathrm{HCO}^{+}$is mainly formed from $\mathrm{C}^{+}$with $\mathrm{CO}^{+}$and $\mathrm{HOC}^{+}$as intermediates via the reactions

$$
\begin{aligned}
& \mathrm{C}^{+}+\mathrm{OH} \rightarrow \mathrm{CO}^{+}+\mathrm{H}, \\
& \mathrm{CO}^{+}+\mathrm{H}_{2} \rightarrow \mathrm{HOC}^{+}+\mathrm{H}, \\
& \mathrm{CO}^{+}+\mathrm{H}_{2} \rightarrow \mathrm{HCO}^{+}+\mathrm{H}, \\
& \mathrm{C}^{+}+\mathrm{H}_{2} \mathrm{O} \rightarrow \mathrm{HOC}^{+}+\mathrm{H},
\end{aligned}
$$

and

$\mathrm{HOC}^{+}+\mathrm{H}_{2} \rightarrow \mathrm{HCO}^{+}+\mathrm{H}_{2}$.

Only a minor amount $(10 \%)$ of $\mathrm{HCO}^{+}$is formed by the reaction between $\mathrm{C}^{+}$and $\mathrm{H}_{2} \mathrm{O}$. If the abundance of parent species in our model photodissociating to form $\mathrm{C}^{+}$is increased, then a minor enhancement of $\mathrm{HCO}^{+}$results:

$\mathrm{HCO}^{+}+\mathrm{e}^{-} \rightarrow \mathrm{CO}+\mathrm{H}$.

The peak abundance of $\mathrm{HCO}^{+}$may also be enhanced by the inclusion of the neutral analogue $\mathrm{HCO}$ as a parent species. A potential route to $\mathrm{HCO}$ in the inner CSE is via the reaction between $\mathrm{H}$ and $\mathrm{CO}$ with a third body (see, e.g., Baulch et al. 2005). The backwards reaction has a large energy barrier $(\approx 8000 \mathrm{~K}$ Baulch et al. 2005), potentially leading to appreciable abundances of $\mathrm{HCO}$. The inclusion of $\mathrm{HCO}$ formation and destruction in models of the inner CSE of O-rich AGB stars are worth investigating in the future.

The electron abundance, which has been discussed in Sect. 3.3, is the most significant factor in destroying many cations. The C-, S-, and Si-bearing parent species contribute most to the formation of electrons depending on the radius. However, the abundances of $\mathrm{C}$ and $\mathrm{Si}$ related species are strongly constrained as discussed in Sect. 2.2 and here we only explore the effect of the uncertainty introduced by the unknown amount of atomic $\mathrm{S}$ among the parent species. In our fiducial model we exclude atomic $S$ as a parent species. However, the shockinduced non-LTE simulation of Cherchneff (2006) suggested that most elemental $\mathrm{S}$ is in atomic form for O-rich AGB stars, which was further confirmed in the latest simulations specifically focussing on IK Tau (Cherchneff, priv. comm.). Since the solar abundance of elemental $\mathrm{S}$ relative to $\mathrm{H}_{2}$ is $2.6 \times 10^{-5}$ (Asplund et al. 2009), and we have $1.1 \times 10^{-5}$ in $\mathrm{SiS}$ in our model (see Table 2), we assume the maximum amount of $S$ in the inner CSE to be $1.5 \times 10^{-5}$. As can be seen in panel c of Fig. B.2, the uncertainty of including or excluding atomic $S$ has a minor effect on the total abundance of electrons, and therefore on $\mathrm{HCO}^{+}$. Interestingly, $\mathrm{NS}$ is found to be sensitive to the initial abundance of atomic S, and may be useful as a diagnostic measurement. More interestingly, as can be seen in panel d of Fig. B.2, both NS and $\mathrm{NO}$ are not affected much by the initial $\mathrm{N}_{2}$ abundance. It is 
hard to detect $\mathrm{N}_{2}$ directly, but it can be indirectly constrained by the abundance of $\mathrm{N}_{2} \mathrm{H}^{+}$, which is mainly formed via the reaction

$\mathrm{N}_{2}+\mathrm{H}_{3}^{+} \rightarrow \mathrm{N}_{2} \mathrm{H}^{+}+\mathrm{H}_{2}$.

At larger radii, e.g., $10^{17} \mathrm{~cm}$, its formation is dominated by

$\mathrm{N}_{2}+\mathrm{He}^{+} \rightarrow \mathrm{N}_{2}^{+}+\mathrm{He}$,

$\mathrm{N}_{2}^{+}+\mathrm{H}_{2} \rightarrow \mathrm{N}_{2} \mathrm{H}^{+}+\mathrm{H}$,

where the $\mathrm{He}^{+}$is generated by cosmic-ray ionization. In all situations, the abundance of $\mathrm{N}_{2} \mathrm{H}^{+}$is directly related to the initial abundance of $\mathrm{N}_{2}$, but this species has not yet been detected in the CSEs of AGB stars.

\section{Concluding remarks}

We simulated the chemistry in O-rich circumstellar envelopes of AGB stars. We have calculated radial abundances suitable for guiding future observational studies and identified the most abundant species. Our model includes the latest gas-phase reaction network and photodissociation processes, including a spectroscopically realistic treatment of $\mathrm{N}_{2}$ and $\mathrm{CO}$ self-shielding. The $\mathrm{H}_{2} \mathrm{O}$ column is found to be too low to self shield. With the proper self-shielding factors, the $\mathrm{N} \rightarrow \mathrm{N}_{2}$ and $\mathrm{C}^{+} \rightarrow \mathrm{CO}$ transitions are shifted outward by factors of 7 and 2, respectively, in the fiducial model representing IK Tau. We also adopt a selection of precursor parent species based on observations and the latest non-LTE model of the interior CSE of IK Tau.

There are few quantitative observational constraints on the molecular abundances in IK Tau, but based on our experience with the C-rich CSE surrounding IRC +10216 (Li et al. 2014) we expect order-of-magnitude accuracy for our predictions of $\mathrm{C}-, \mathrm{N}-, \mathrm{O}-$, and Si-bearing molecules. The reaction networks for less abundant elements ( $\mathrm{S}, \mathrm{P}, \mathrm{Cl}$, and $\mathrm{F}$ ) are not as well known, but significant abundances of their host molecules are predicted by our model. Many molecules have peak abundance between $10^{16}$ to $10^{17} \mathrm{~cm}$ from the star where the ionization and dissociation of parent species provides a source of radicals but where molecules are still somewhat shielded from interstellar radiation. Key steps in the intervening chemistry are hydrogen abstraction of radical ions with $\mathrm{H}_{2}$ and the production of neutral fragments by dissociative recombination and the ultimate destruction of all molecules by the ISRF.

We find chemical links between potentially observable daughter molecules and the abundances of parent species in the inner CSE. Observationally derived upper limits for the amount of $\mathrm{C}_{2} \mathrm{H}$ and $\mathrm{CH}$ in IK Tau provide a low constraint on the relative abundance of the potential parent $\mathrm{CH}_{4},<2.5 \times 10^{-6}$. We also find that observations of $\mathrm{NS}$ and $\mathrm{N}_{2} \mathrm{H}^{+}$could provide indirect constraints on the parent abundances of atomic $\mathrm{S}$ and $\mathrm{N}_{2}$.

Our modeled molecular abundances are also sensitive to the assumed physical parameters of the CSE. We find that the stellar mass-loss rate, which is usually not straightforward to obtain from observations, has a large impact on the calculated abundances. On the other hand, variations in the envelope expansion velocity and cosmic-ray ionization rate are not as significant (see Appendix A).

Many of the predictions of our models (both overall abundances and peak abundance radii) can be tested directly by spatially resolved observations with ALMA in the near future.

Acknowledgements. X. Li thanks T.J.M. for his hospitality during a two-week visit to Queen's University Belfast (QUB) in 2014, and thanks Prof. Xander Tielens and Prof. Leen Decin for the stimulating discussions. X. Li would also like to thank Drs. Jessica Edwards and Robin Pulliam for the discussion on $\mathrm{HCO}^{+}$in IK Tau. Astrochemistry in Leiden is supported by the Netherlands Research School for Astronomy (NOVA), by a Spinoza grant and grant 648.000.002 from the Netherlands Organisation for Scientific Research (NWO), and by the European Community's Seventh Framework Programme FP7/2007-2013 under grant agreements 291141 (CHEMPLAN) and 238258 (LASSIE). Astrophysics at QUB is supported by a grant from the STFC. C.W. acknowledges support from NWO (program number 639.041.335). X. Li acknowledges support from EACOA Fellowship.

\section{References}

Agúndez, M., Cernicharo, J., \& Guélin, M. 2010a, ApJ, 724, L133 Agúndez, M., Cernicharo, J., Guélin, M., et al. 2010b, A\&A, 517, L2

Ajello, J. M., James, G. K., Franklin, B. O., \& Shemansky, D. E. 1989, Phys. Rev. A, 40, 3524

Asplund, M., Grevesse, N., Sauval, A. J., \& Scott, P. 2009, ARA\&A, 47, 481

Baulch, D. L., Bowman, C. T., Cobos, C. J., et al. 2005, J. Phys. Chem. Ref. Data, 34, 757

Bergeat, A., Calvo, T., Dorthe, G., \& Loison J.-C. 1999, J. Phys. Chem. A, 103, 6360

Bethell, T., \& Bergin, E. 2009, Science, 326, 1675

Bohlin, R. C., Savage, B. D., \& Drake, J. F. 1978, ApJ, 224, 132

Bujarrabal, V., Fuente, A., \& Omont, A. 1994, A\&A, 285, 247

Cernicharo, J., Guélin, M., \& Kahane, C. 2000, A\&AS, 142, 181

Cherchneff, I. 2006, A\&A, 456, 1001

Cherchneff, I. 2012, A\&A, 545, A12

Cho, S.-H., \& Ukita, N. 1998, AJ, 116, 2495

Cordiner, M. A., \& Millar, T. J. 2009, ApJ, 697, 68

Danilovich, T., Bergman, P., Justtanont, K., et al. 2014, A\&A, 569, A76

De Beck, E., Decin, L., de Koter, A., et al. 2010, A\&A, 523, A18

De Beck, E., Lombaert, R., Agúndez, M., et al. 2012, A\&A, 539, A108

De Beck, E., Kamiński, T., Patel, N. A., et al. 2013, A\&A, 558, A132

De Luca, M., Gupta, H., Neufeld, D., et al. 2012, ApJ, 751, L37

Decin, L. 2012, Adv. Space Res., 50, 843

Decin, L., Cherchneff, I., Hony, S., et al. 2008, A\&A, 480, 431

Decin, L., Agúndez, M., Barlow, M. J., et al. 2010a, Nature, 467, 64

Decin, L., De Beck, E., Brünken, S., et al. 2010b, A\&A, 516, A69

Decin, L., Justtanont, K., De Beck, E., et al. 2010c, A\&A, 521, L4

Draine, B. T. 1978, ApJS, 36, 595

Duari, D., Cherchneff, I., \& Willacy, K. 1999, A\&A, 341, L47

Fuchs, G. W., Cuppen, H. M., Ioppolo, S., et al. 2009, A\&A, 505, 629

Garrod, R. T., \& Herbst, E. 2006, A\&A, 457, 927

Geppert, W. D., Hamberg, M., Thomas, R. D., et al. 2006, Faraday Discuss., 133, 177

Gerin, M., de Luca, M., Black, J., et al. 2010, A\&A, 518, L110

Gerin, M., de Luca, M., Lis, D. C., et al. 2013, J. Phys. Chem. A, 117, 10018

Gielen, C., Cami, J., Bouwman, J., Peeters, E., \& Min, M. 2011, A\&A, 536, A54

Glassgold, A. E., \& Huggins, P. J. 1983, MNRAS, 203, 517

Gobrecht, D., Cherchneff, I., Sarangi, A., Plane, J. M. C., \& Bromley, S. T. 2016, A\&A, 585, A6

Guélin, M., Neininger, N., Lucas, R., \& Cernicharo, J. 1999, in The Physics and Chemistry of the Interstellar Medium, eds. V. Ossenkopf, J. Stutzki, \& G. Winnewisser, 326

Heays, A. N., Dickenson, G. D., Salumbides, E. J., et al. 2011, J. Chem. Phys., 135

Heays, A. N., Visser, R., Gredel, R., et al. 2014, A\&A, 562, A61

Helm, H., Hazell, I., \& Bjerre, N. 1993, Phys. Rev. A, 48, 2762

Herwig, F. 2005, ARA\&A, 43, 435

Iben, Jr., I., \& Renzini, A. 1983, ARA\&A, 21, 271

Jansen, D. J., Spaans, M., Hogerheijde, M. R., \& van Dishoeck, E. F. 1995a, A\&A, 303, 541

Jansen, D. J., van Dishoeck, E. F., Black, J. H., Spaans, M., \& Sosin, C. 1995b, A\&A, 302, 223

Justtanont, K., Khouri, T., Maercker, M., et al. 2012, A\&A, 537, A144

Kim, H., Wyrowski, F., Menten, K. M., \& Decin, L. 2010, A\&A, 516, A68

Kwon, Y.-J., \& Suh, K.-W. 2012, J. Korean Astron. Soc., 45, 139

Lee, H.-H., Herbst, E., Pineau des Forets, G., Roueff, E., \& Le Bourlot, J. 1996, A\&A, 311, 690

Lewis, B. R., Gibson, S. T., Zhang, W., Lefebvre-Brion, H., \& Robbe, J.-M 2005, J. Chem. Phys., 122, 144302

Lewis, B. R., Baldwin, K. G. H., Sprengers, J. P., et al. 2008a, J. Chem. Phys., 129,164305

Lewis, B. R., Heays, A. N., Gibson, S. T., Lefebvre-Brion, H., \& Lefebvre, R. 2008b, J. Chem. Phys., 129, 164306

Li, X., Heays, A. N., Visser, R., et al. 2013, A\&A, 555, A14 
Li, X., Millar, T. J., Walsh, C., Heays, A. N., \& van Dishoeck, E. F. 2014, A\&A, 568, A111

Lis, D. C., Phillips, T. G., Goldsmith, P. F., et al. 2010, A\&A, 521, L26

Loup, C., Forveille, T., Omont, A., \& Paul, J. F. 1993, A\&AS, 99, 291

Maercker, M., Schöier, F. L., Olofsson, H., Bergman, P., \& Ramstedt, S. 2008, A\&A, 479, 779

Maercker, M., Schöier, F. L., Olofsson, H., et al. 2009, A\&A, 494, 243

Maercker, M., Mohamed, S., Vlemmings, W. H. T., et al. 2012, Nature, 490, 232

Marvel, K. B. 2005, AJ, 130, 261

McElroy, D., Walsh, C., Markwick, A. J., et al. 2013, A\&A, 550, A36

Menten, K. M., Wyrowski, F., Alcolea, J., et al. 2010, A\&A, 521, L7

Milam, S. N., Apponi, A. J., Woolf, N. J., \& Ziurys, L. M. 2007, ApJ, 668, L131

Millar, T. J., Herbst, E., \& Bettens, R. P. A. 2000, MNRAS, 316, 195

Morris, M., \& Jura, M. 1983, ApJ, 264, 546

Ndome, H., Hochlaf, M., Lewis, B. R., et al. 2008, J. Chem. Phys., 129, 164307

Neufeld, D. A., Roueff, E., Snell, R. L., et al. 2012, ApJ, 748, 37

Pilbratt, G. L., Riedinger, J. R., Passvogel, T., et al. 2010, A\&A, 518, L1

Polehampton, E. T., Menten, K. M., van der Tak, F. F. S., \& White, G. J. 2010, A\&A, 510, A80

Pulliam, R. L., Edwards, J. L., \& Ziurys, L. M. 2011, ApJ, 743, 36

Quan, D., Herbst, E., Osamura, Y., \& Roueff, E. 2010, ApJ, 725, 2101

Quintana-Lacaci, G., Agúndez, M., Cernicharo, J., et al. 2013, A\&A, 560, L2

Ramstedt, S., Schöier, F. L., Olofsson, H., \& Lundgren, A. A. 2006, A\&A, 454, L103

Ramstedt, S., Schöier, F. L., \& Olofsson, H. 2009, A\&A, 499, 515

Ramstedt, S., Maercker, M., Olofsson, G., Olofsson, H., \& Schöier, F. L. 2011, A\&A, 531, A148

Savage, B. D., \& Mathis, J. S. 1979, ARA\&A, 17, 73

Schöier, F. L., Bast, J., Olofsson, H., \& Lindqvist, M. 2007, A\&A, 473, 871

Schöier, F. L., Maercker, M., Justtanont, K., et al. 2011, A\&A, 530, A83

Schöier, F. L., Ramstedt, S., Olofsson, H., et al. 2013, A\&A, 550, A78
Spelsberg, D., \& Meyer, W. 2001, J. Chem. Phys., 115, 6438

Sprengers, J. P., Ubachs, W., Johansson, A., et al. 2004, J. Chem. Phys., 120, 8973

Stark, G., Lewis, B. R., Heays, A. N., et al. 2008, J. Chem. Phys., 128, 114302

Sternberg, A., \& Dalgarno, A. 1995, ApJS, 99, 565

Szczerba, R., Schmidt, M. R., \& Pulecka, M. 2007, Balt. Astron., 16, 134

Tenenbaum, E. D., \& Ziurys, L. M. 2010, ApJ, 712, L93

Tenenbaum, E. D., Dodd, J. L., Milam, S. N., Woolf, N. J., \& Ziurys, L. M. 2010, ApJ, 720, L102

van Dishoeck, E. F. 1988, in Rate Coefficients in Astrochemistry, eds. T. J. Millar, \& D. A. Williams (Dordrecht: Kluwer Academic Publishers), 49

Velilla Prieto, L., Sánchez Contreras, C., Cernicharo, J., et al. 2015, A\&A, 575, A84

Visser, R., van Dishoeck, E. F., \& Black, J. H. 2009, A\&A, 503, 323

Vlemmings, W. H. T., Ramstedt, S., Rao, R., \& Maercker, M. 2012, A\&A, 540, L3

Wakelam, V., Smith, I. W. M., Herbst, E., et al. 2010, Space Sci. Rev., 156, 13

Wakelam, V., Smith, I. W. M., Loison, J.-C., et al. 2013, unpublished [arXiv: 1310.4350$]$

Watanabe, N., \& Kouchi, A. 2002, ApJ, 571, L173

Willacy, K., \& Millar, T. J. 1997, A\&A, 324, 237

Willems, F. J., \& de Jong, T. 1986, ApJ, 309, L39

Woods, P. M., Schöier, F. L., Nyman, L.-Å., \& Olofsson, H. 2003, A\&A, 402, 617

Wu, M., \& Fehlner, T. 1975, Chem. Phys. Lett., 36, 114

Zack, L. N., Halfen, D. T., \& Ziurys, L. M. 2011, ApJ, 733, L36

Ziurys, L. M. 2006, PNAS, 103, 12274

Ziurys, L. M., Milam, S. N., Apponi, A. J., \& Woolf, N. J. 2007, Nature, 447, 1094

Ziurys, L. M., Tenenbaum, E. D., Pulliam, R. L., Woolf, N. J., \& Milam, S. N. 2009, ApJ, 695, 1604 


\section{Appendix A: An extended investigation of the CSE species}

The chemical evolution of molecular species in an expanding CSE naturally depends on the physical structure of the AGB star and its envelope. In particular, the mass-loss rate plays an important role in the distribution of the molecules in the outer envelope, but large uncertainties always exist in its measurement. Starting from our physico-chemical model of IK Tau, we explore here the chemistry of a few interesting species over a range of typical mass-loss rates and gas expansion velocities, $10^{-8}-10^{-4} M_{\odot} \mathrm{yr}^{-1}$ (Kwon \& Suh 2012) and 5-40 $\mathrm{km} \mathrm{s}^{-1}$, respectively. The predicted trends are relevant to O-rich AGB stars in general.

The effect of altering the mass-loss rate of an AGB star on gas density, visual extinction, and the abundance of molecular species (both parent and daughter) is studied assuming a fixed envelope expansion velocity, $20 \mathrm{~km} \mathrm{~s}^{-1}$, whereas the dependence on expansion velocity is studied by assuming a fixed mass-loss rate of $10^{-5} M_{\odot} \mathrm{yr}^{-1}$. Figure A.1 shows that the photodissociation region is shifted outwards for increasing mass-loss rate, whereas the peak abundances of most neutral species, e.g., $\mathrm{CN}$ and NO, are less affected. This implies that peak radii of daughter species retrieved from observations may give information about the mass-loss rate. In contrast, the peak abundances of cations, e.g., $\mathrm{N}_{2} \mathrm{H}^{+}$and $\mathrm{HCO}^{+}$, are decreased and the peak radius moves outwards with increasing mass-loss rate. We note that the decrease in peak abundance is more apparent for $\mathrm{HCO}^{+}$ than $\mathrm{N}_{2} \mathrm{H}^{+}$and for the highest mass-loss rate, $10^{-4} M_{\odot} \mathrm{yr}^{-1}$, the peak abundance for the latter begins to increase as the peak is pushed outwards. This effect on cations is due to the physical structure of the CSE and not to their enhanced chemical destruction. Their destruction is dominated by dissociative recombination with electrons and the electron density decreases significantly with increasing mass loss.

Total column densities of four molecular species are shown in Fig. A.2 as functions of mass-loss rate and gas expansion velocity. This figure, together with the upper left panel of Fig. A.1, shows that the dependence of the chemistry on the expansion velocity is negligible within usual observational uncertainties. Trends for all parent species are similar to those plotted for $\mathrm{H}_{2} \mathrm{O}$, whose column density increases with increasing mass-loss rate. However, the modeled column density trends for daughter species are quite variable, e.g., the behavior of $\mathrm{OH}$ is similar to that of its parent species $\mathrm{H}_{2} \mathrm{O}$, whereas $\mathrm{H}_{2} \mathrm{Cl}^{+}$exhibits a very different trend. According to this study, the upper limit of the $\mathrm{HCO}^{+}$column density in an O-rich envelope is $5 \times 10^{11} \mathrm{~cm}^{-2}$. 

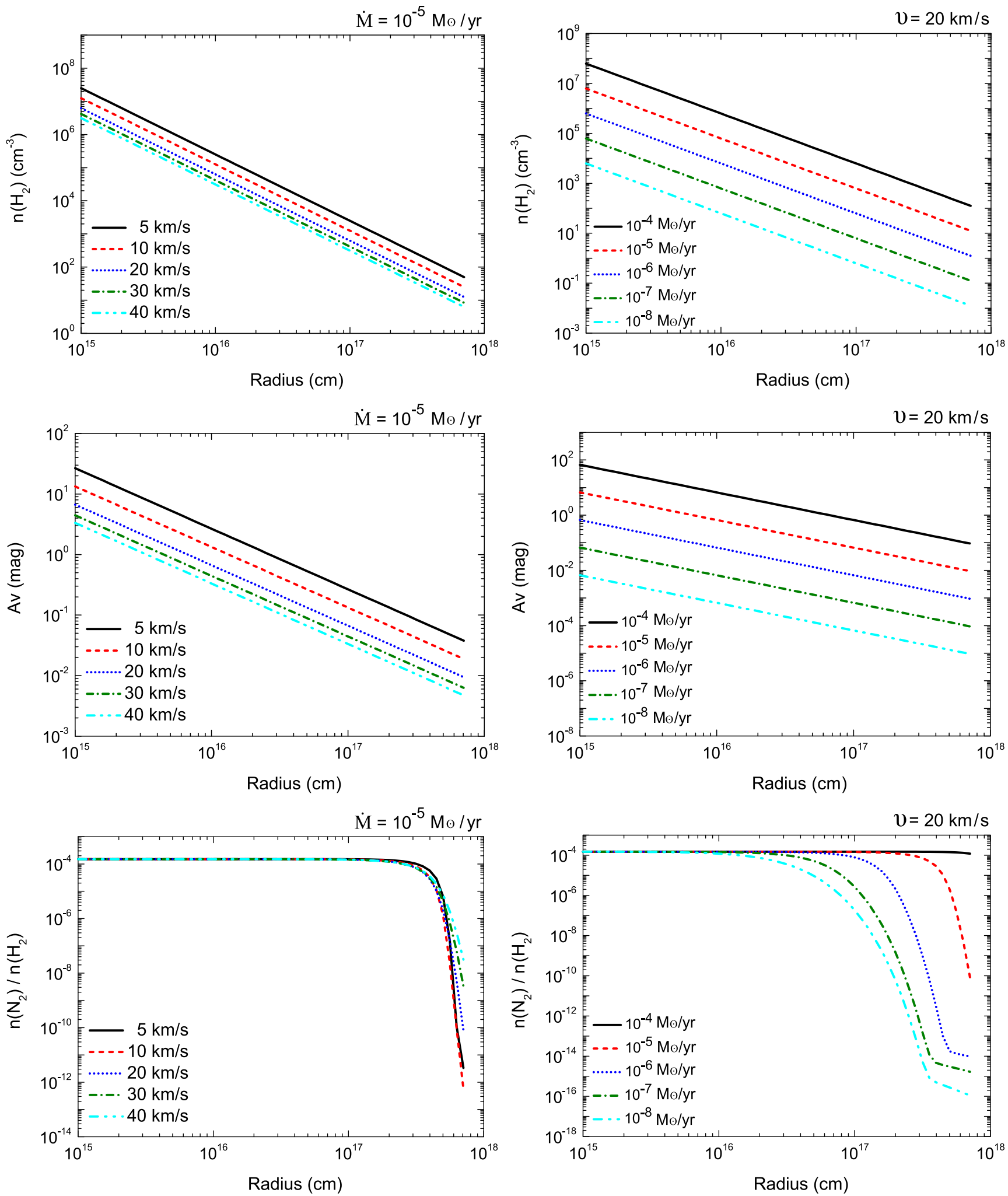

Fig. A.1. Effects of envelope expansion velocity and mass-loss rate on gas density, visual extinction, and distributions of species in the outer layers of O-rich AGB stars, part A. 
X. Li et al.: Chemistry of molecules in the CSEs of O-rich AGB stars
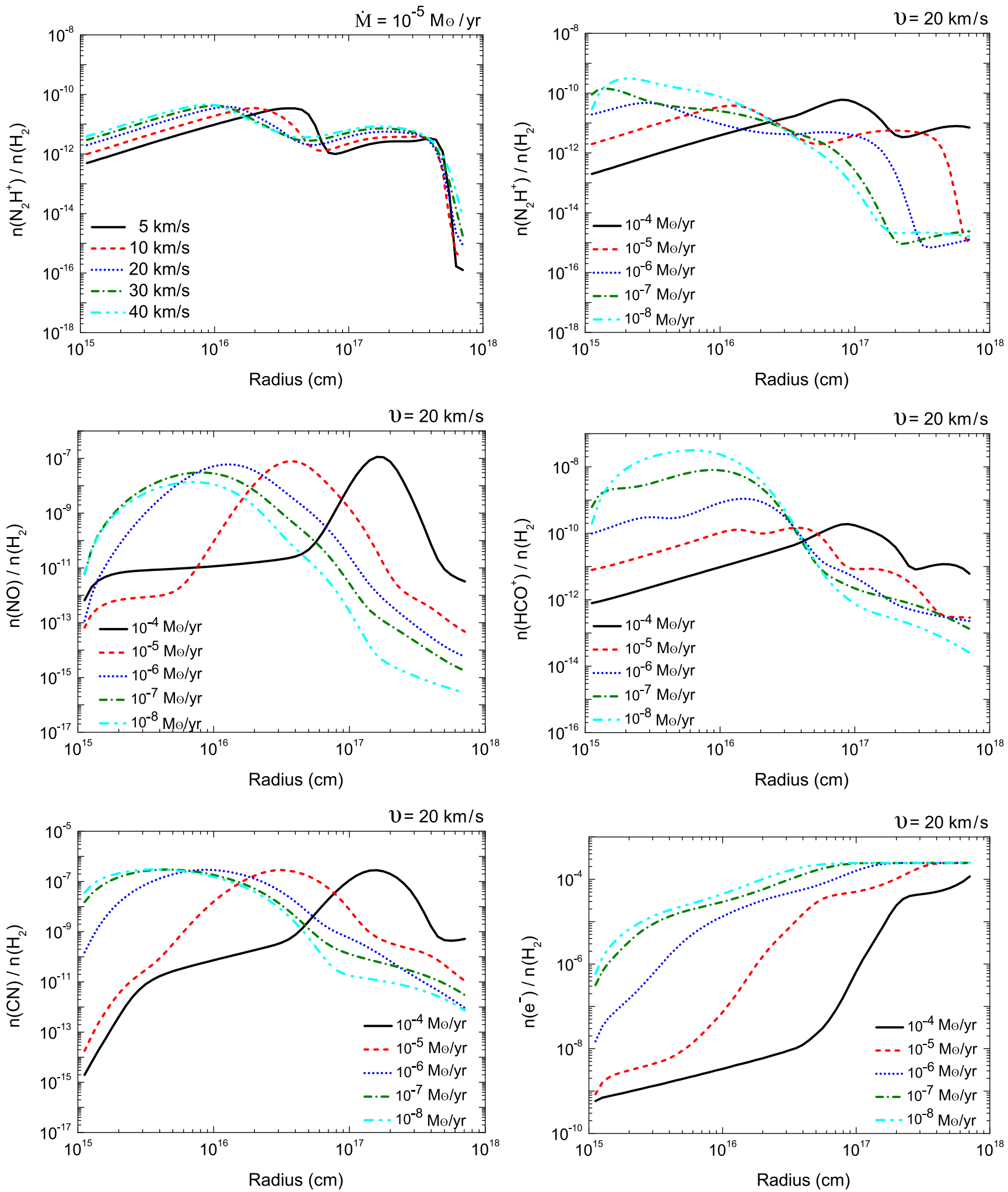

Fig. A.1. continued, part B. 
A\&A 588, A4 (2016)
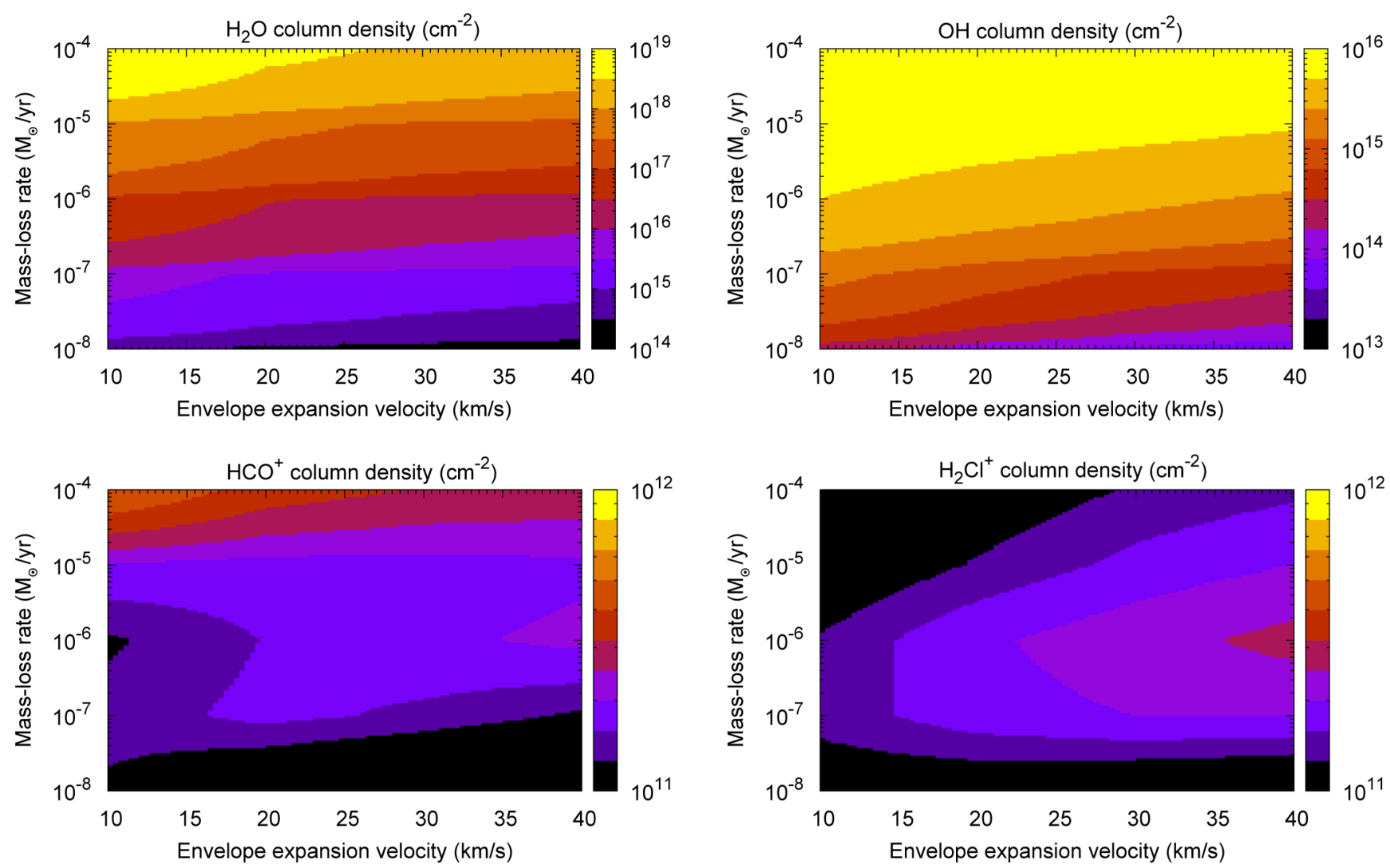

Fig. A.2. Total column densities for a sample of species in the CSE of O-rich AGB stars, as a function of mass-loss rate and envelope expansion velocity. 
$\mathrm{X}$. Li et al.: Chemistry of molecules in the CSEs of O-rich AGB stars

Appendix B: Additional figures

(a)

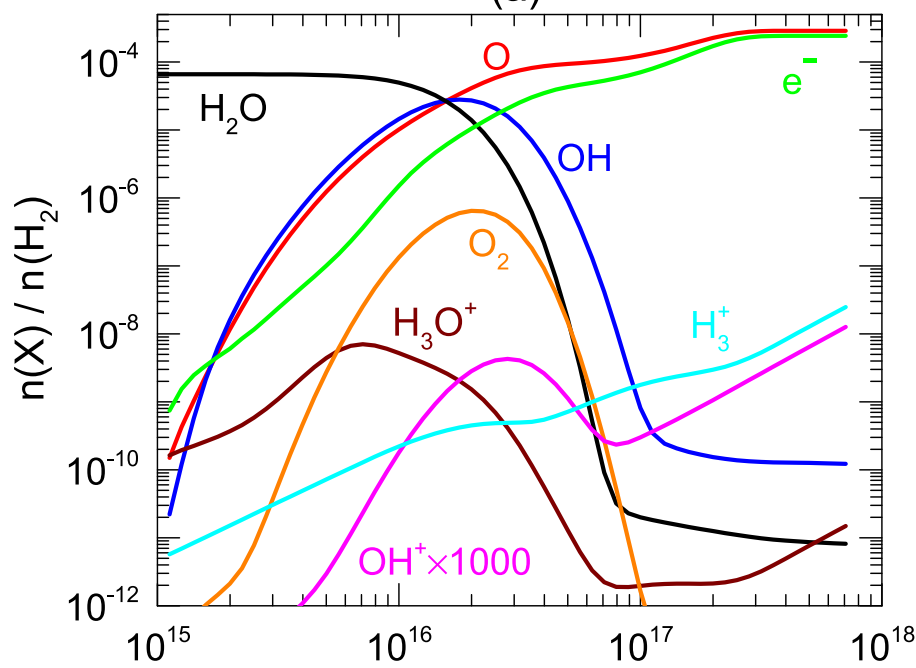

(c)

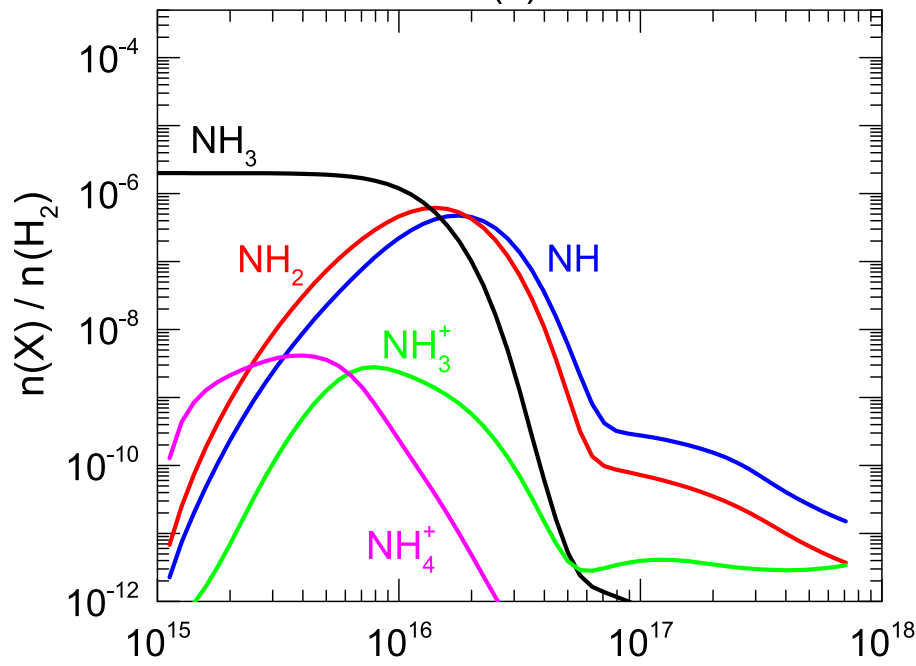

(e)

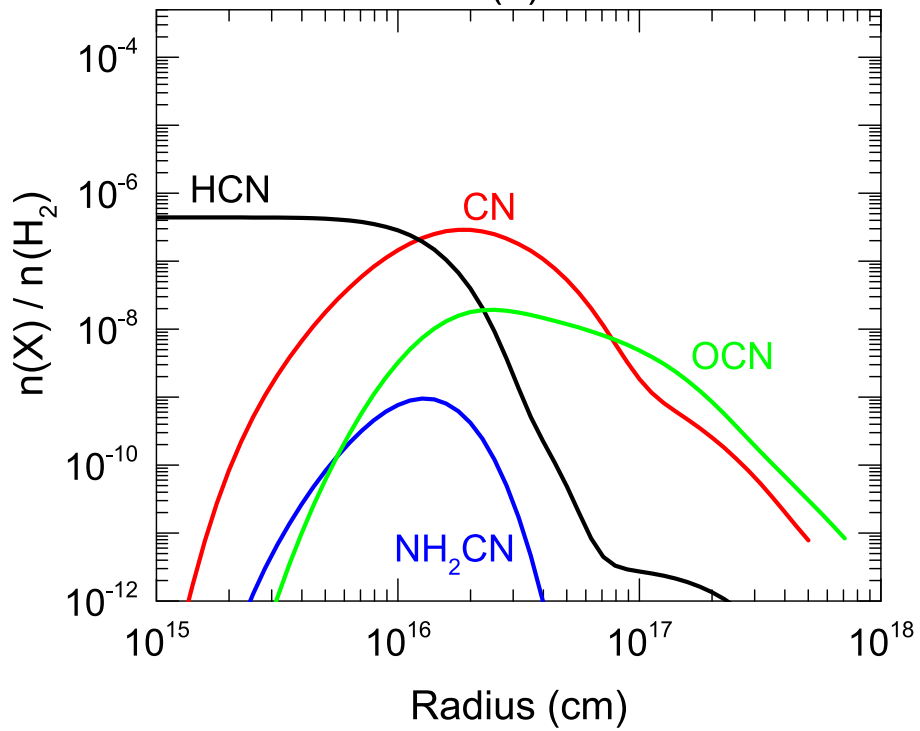

(b)

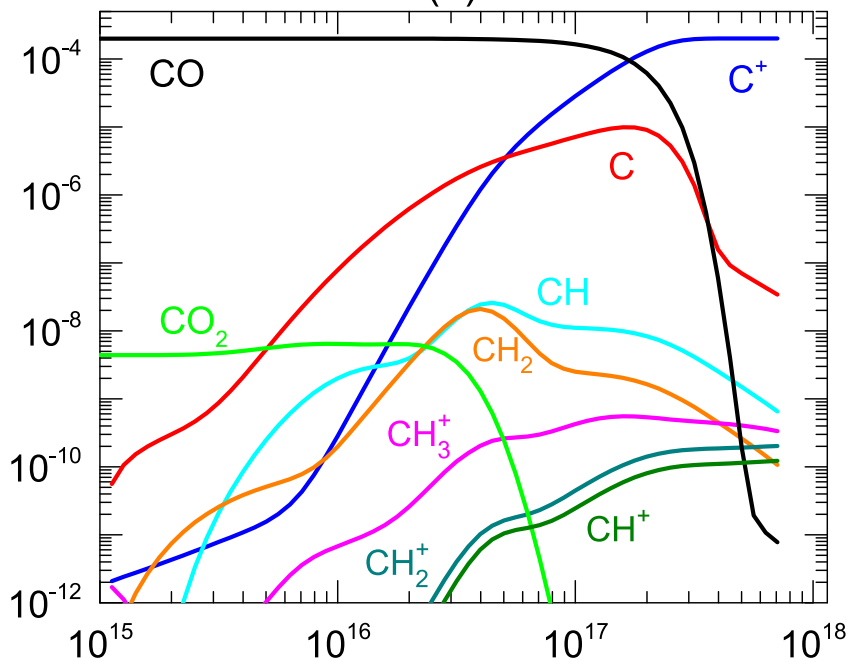

(d)

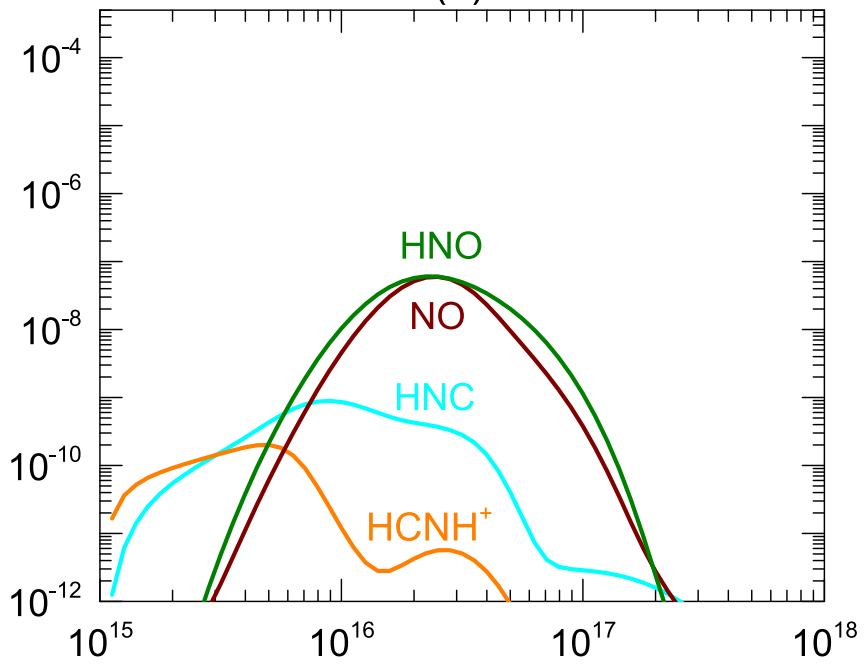

(f)

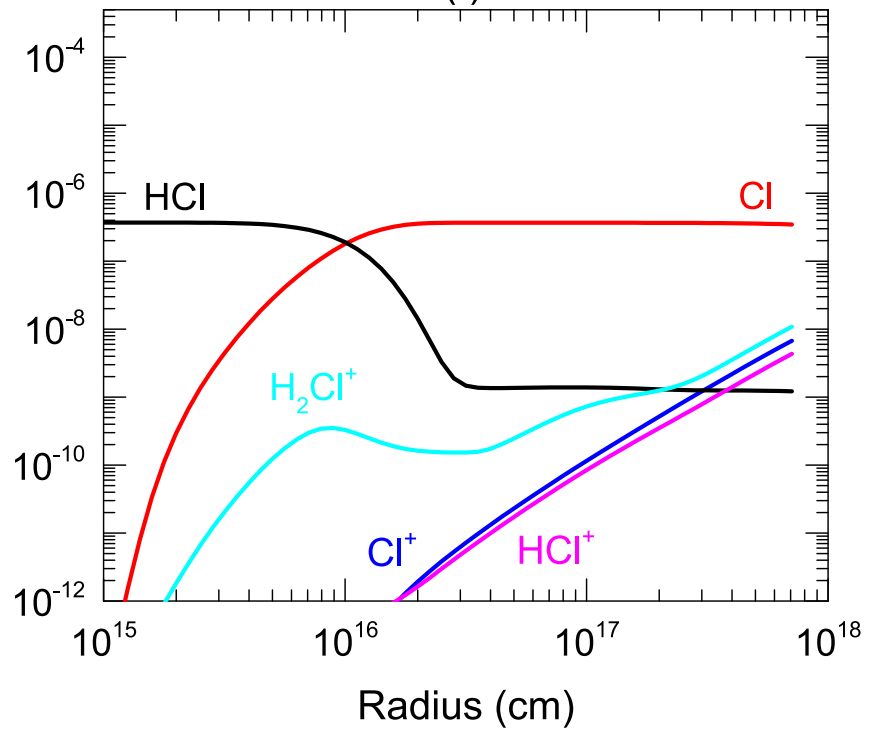

Fig. B.1. Fractional abundances, relative to $\mathrm{H}_{2}$, of the most abundant species in the outer CSE of the O-rich AGB star, IK Tau. 
(g)

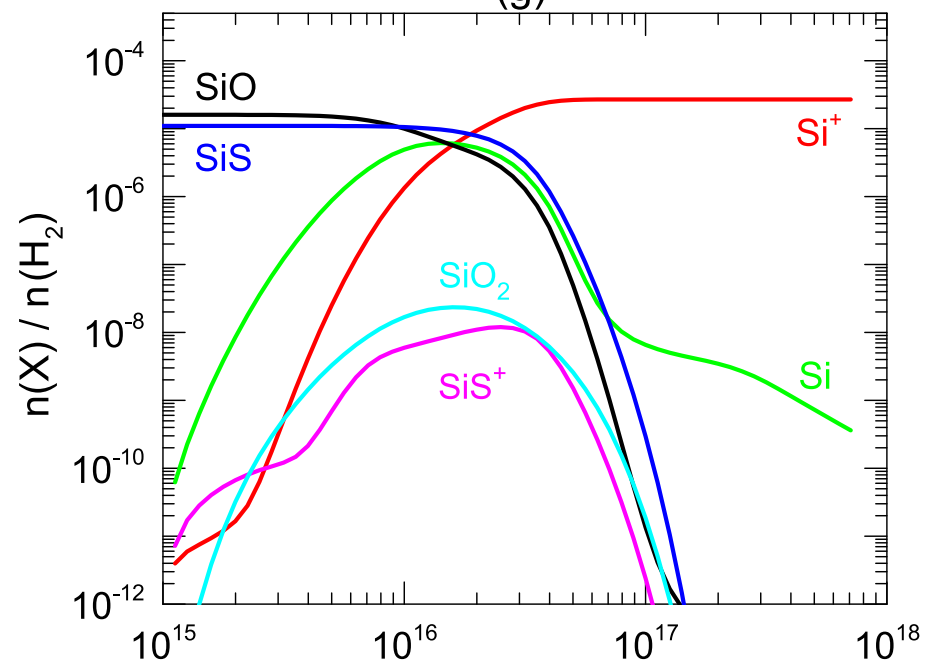

(i)

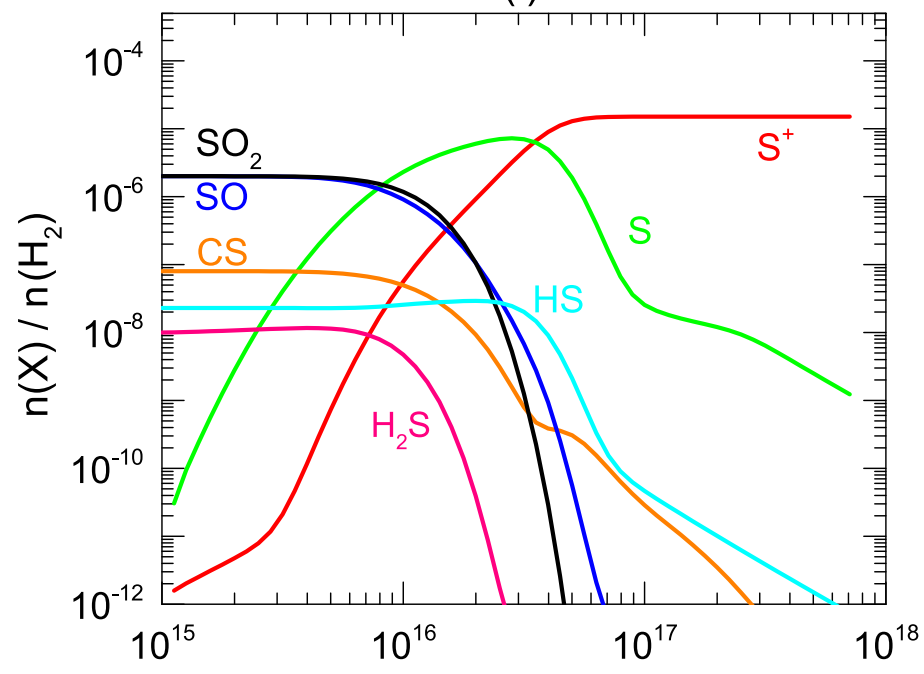

(k)

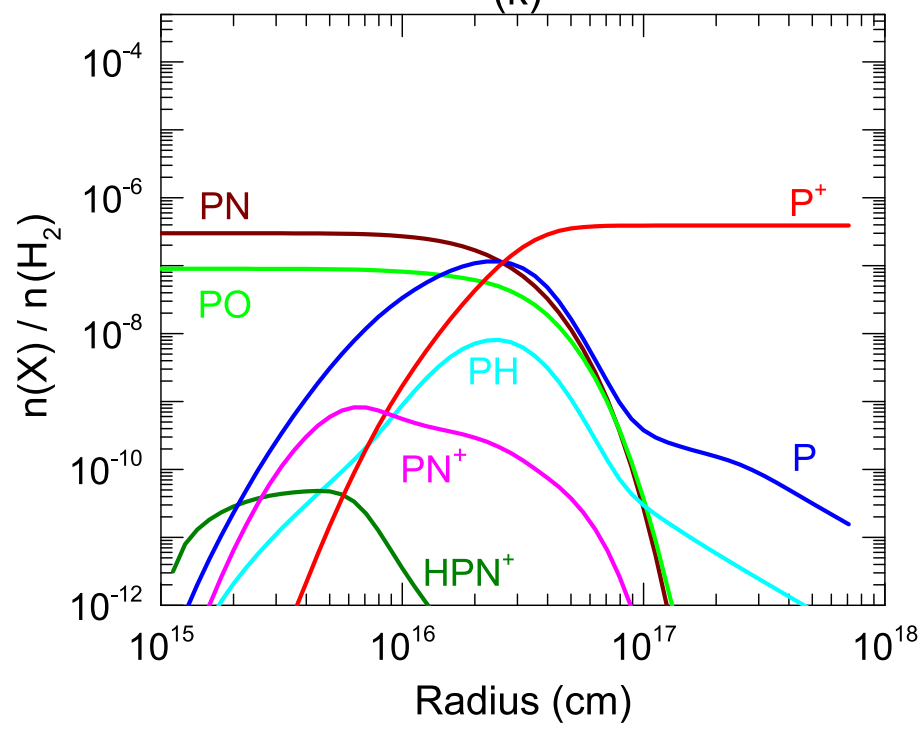

(h)

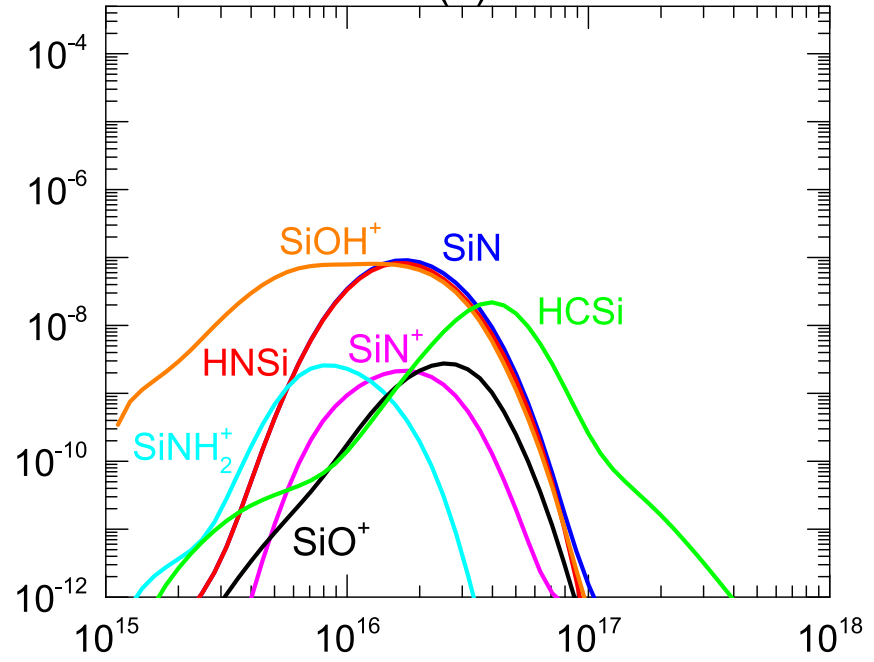

(j)

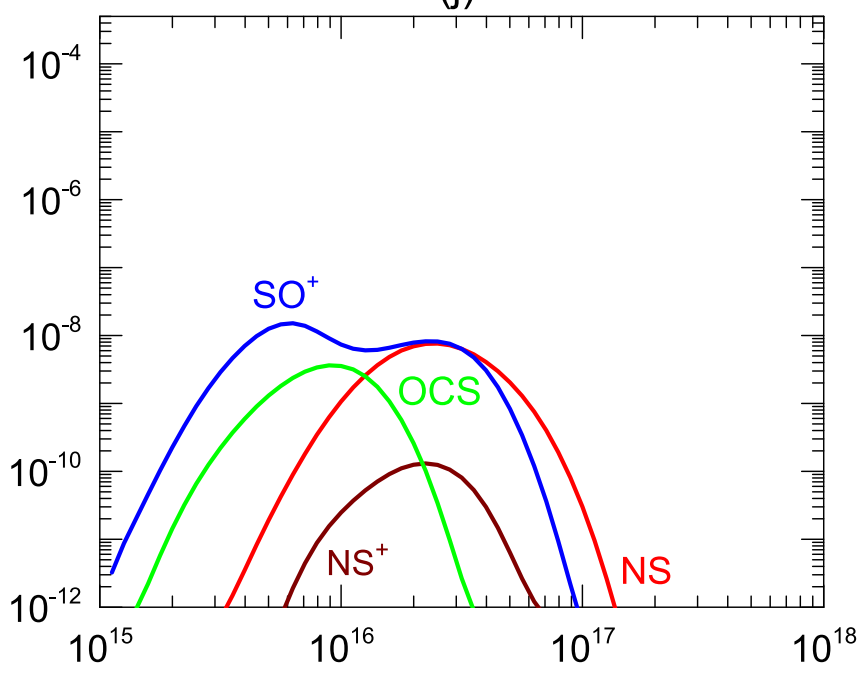

(I)

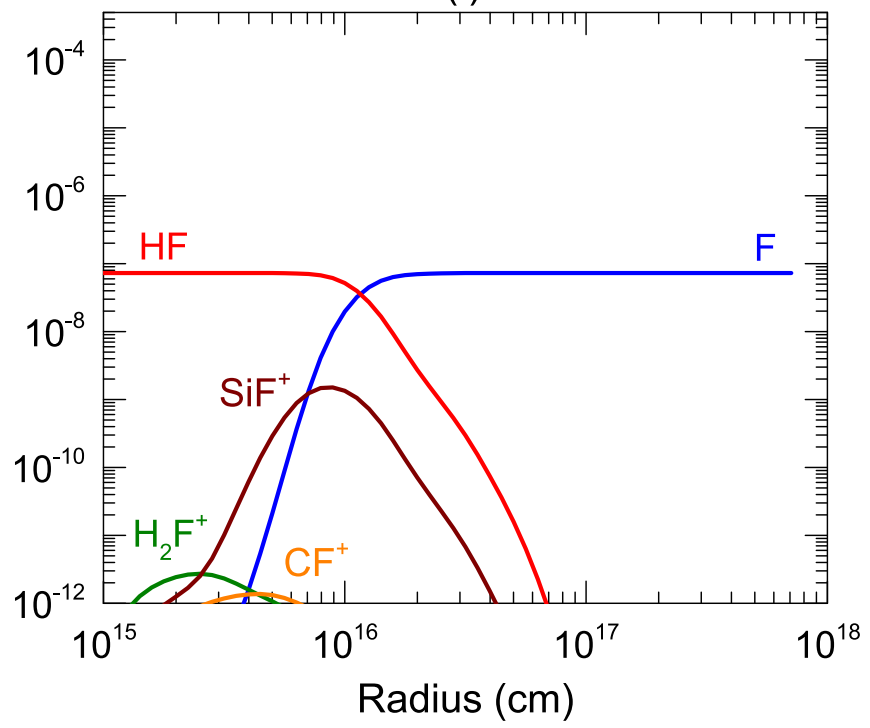

Fig. B.1. continued. 
$\mathrm{X}$. Li et al.: Chemistry of molecules in the CSEs of O-rich AGB stars

(a)

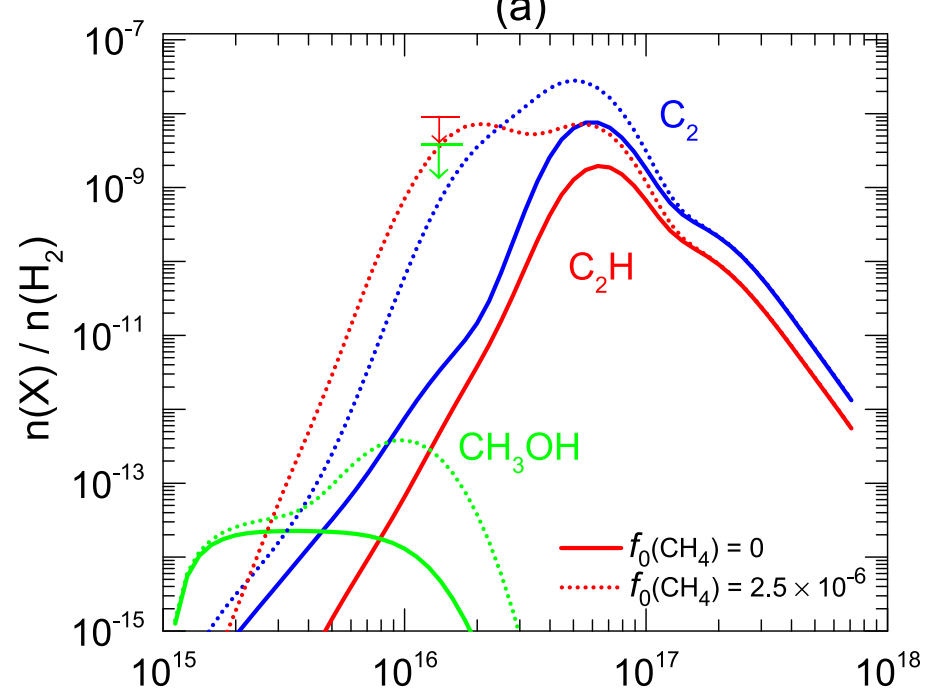

(c)

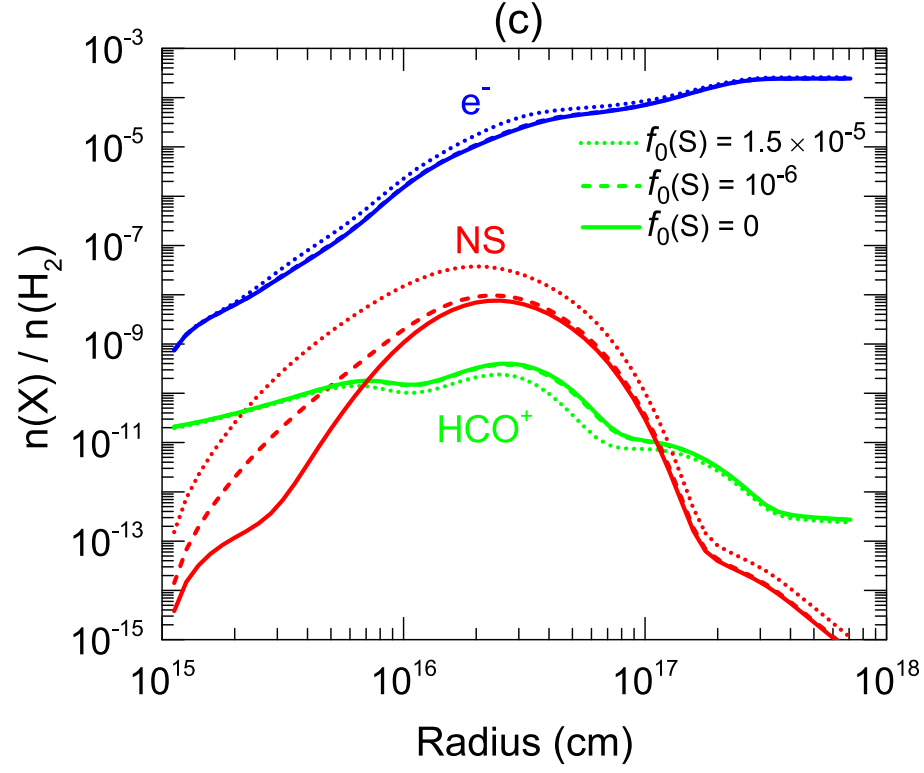

(b)

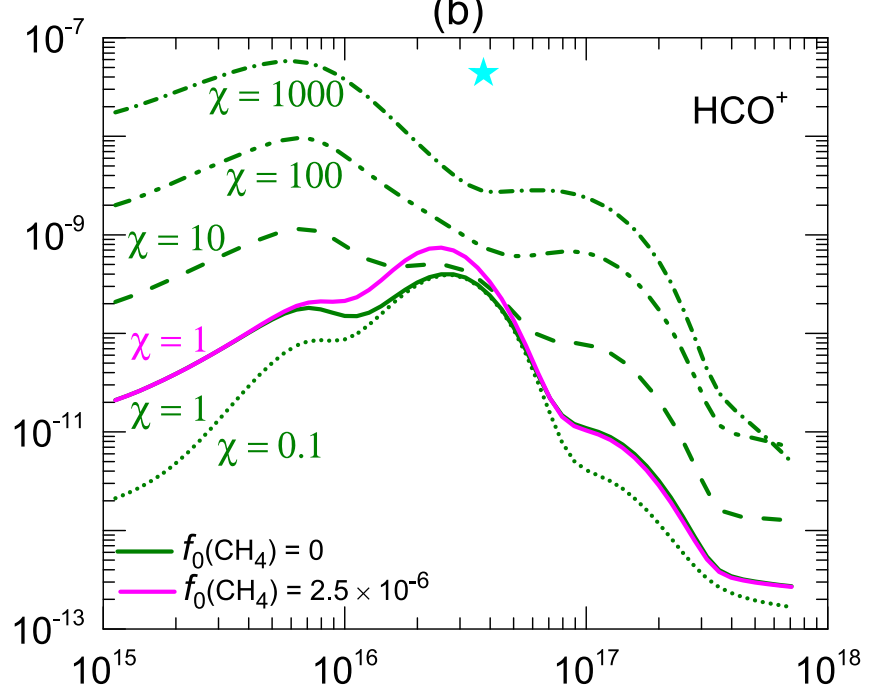

(d)

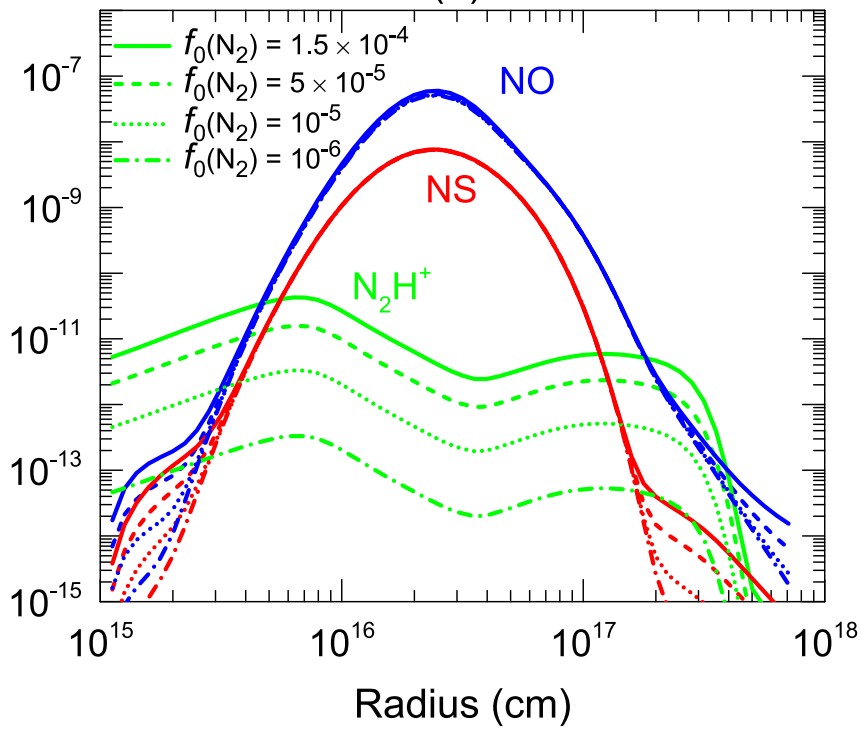

Fig. B.2. Fractional abundances of some daughter species as a function of radius, calculated using different initial fractions of $\mathrm{CH}_{4}$ (panels a) and $\mathbf{b})$ ), atomic $\mathrm{S}$ (panel $\mathbf{c}$ )), and $\mathrm{N}_{2}$ (panel d)), in addition to various cosmic-ray rates. In panel a): the arrows indicate the upper limits of $\mathrm{C}_{2} \mathrm{H}$ (red) and $\mathrm{CH}_{3} \mathrm{OH}$ (green) deduced in the observational work of Marvel (2005) where the peak radii of these species was assumed to be $1.5 \times 10^{16} \mathrm{~cm}$. In panel b): $\chi$ is the scaling factor of the cosmic-ray ionization rate and the star indicates the observational result from Pulliam et al. (2011). 